\title{
Analyse de la cohabitation spatiale des communautés immigrantes avec les francophones et les anglophones de la région métropolitaine de Montréal Analysis of spatial cohabitation between immigrant communities and Francophones and Anglophones in the Montreal metropolitan area
}

\author{
Guillaume Marois, Sébastien Lord et Alain Bélanger
}

Volume 46, numéro 1, printemps 2017

URI : https://id.erudit.org/iderudit/1043298ar

DOI : https://doi.org/10.7202/1043298ar

Aller au sommaire du numéro

Éditeur(s)

Association des démographes du Québec

ISSN

1705-1495 (numérique)

Découvrir la revue

Citer cet article

Marois, G., Lord, S. \& Bélanger, A. (2017). Analyse de la cohabitation spatiale des communautés immigrantes avec les francophones et les anglophones de la région métropolitaine de Montréal. Cahiers québécois de démographie, 46(1), 129-156. https://doi.org/10.7202/1043298ar

\section{Résumé de l'article}

Au cours des $20^{\mathrm{e}}$ et $21^{\mathrm{e}}$ siècles, la région métropolitaine de Montréal a connu plusieurs vagues successives d'immigration provenant de plusieurs pays et abrite aujourd'hui nombre de communautés d'origines diverses. Si la théorie de l'assimilation spatiale prévoit que peu à peu, les immigrants tendent à avoir des comportements comparables à ceux des natifs en matière de localisation résidentielle, ce processus peut suivre différentes dynamiques dans la région métropolitaine de Montréal, puisqu'elle est historiquement peuplée de deux grandes communautés natives, les francophones et les anglophones, qui ont chacun des comportements résidentiels distincts. L'objectif de cet article est de mesurer et d'analyser la cohabitation résidentielle des personnes issues de l'immigration avec les communautés d'accueil francophone et anglophone. La population étudiée est sélectionnée dans l'Enquête nationale auprès des ménages de 2011 et est constituée de la population vivant dans un ménage dont le principal soutien est soit lui-même né à l'étranger, soit a un parent né à l'étranger. Pour chacune des quinze plus importantes communautés immigrantes, nous avons construit deux modèles de régressions ayant comme variables dépendantes respectivement la proportion de francophones de $3^{\mathrm{e}}$ génération vivant dans le quartier et la proportion d'anglophones de $3^{\mathrm{e}}$ génération, de manière à calculer des indices d'interaction standardisés selon une série de variables indépendantes (âge, sexe, durée de résidence, scolarité, revenu et connaissances linguistiques). Il en ressort que dans l'ensemble, l'assimilation spatiale des communautés immigrantes ne se fait pas en référence aux francophones majoritaires, mais plutôt en référence à la minorité anglophone. Une forte hétérogénéité s’observe toutefois entre les communautés et au sein de celles-ci.
Tous droits réservés (C) Association des démographes du Québec, 2017
Ce document est protégé par la loi sur le droit d'auteur. L’utilisation des services d’Érudit (y compris la reproduction) est assujettie à sa politique d'utilisation que vous pouvez consulter en ligne. 
Cahiers québécois de démographie

Vol. 46, $\mathrm{n}^{\circ}$ 1, printemps 2017, p. 129-156

\title{
Analyse de la cohabitation spatiale des communautés immigrantes avec les francophones et les anglophones de la région métropolitaine de Montréal
}

\author{
GUILLAUME MAROIS ${ }^{\star}$, SÉBASTIEN LORD $^{\star *}$ ET ALAIN BÉLANGER***
}

\begin{abstract}
RÉSUMÉ
Au cours des $20^{\mathrm{e}}$ et $21^{\mathrm{e}}$ siècles, la région métropolitaine de Montréal a connu plusieurs vagues successives d'immigration provenant de plusieurs pays et abrite aujourd'hui nombre de communautés d'origines diverses. Si la théorie de l'assimilation spatiale prévoit que peu à peu, les immigrants tendent à avoir des comportements comparables à ceux des natifs en matière de localisation résidentielle, ce processus peut suivre différentes dynamiques dans la région métropolitaine de Montréal, puisqu'elle est historiquement peuplée de deux grandes communautés natives, les francophones et les anglophones, qui ont chacun des comportements résidentiels distincts. L'objectif de cet article est de mesurer et d'analyser la cohabitation résidentielle des personnes issues de l'immigration avec les communautés d'accueil francophone et anglophone. La population étudiée est sélectionnée dans l'Enquête nationale auprès des ménages de 2011 et est constituée de la population vivant dans un ménage dont le principal soutien est soit lui-même né à l'étranger, soit a un parent né à l'étranger. Pour chacune des quinze plus importantes communautés immigrantes, nous avons construit deux modèles de régressions ayant comme variables dépendantes respectivement la proportion de francophones de $3^{\mathrm{e}}$ génération vivant dans le quartier et la proportion d'anglophones de $3^{e}$ génération, de manière à calculer des indices d'interaction standardisés selon une série de variables indépendantes (âge, sexe, durée de résidence, scolarité, revenu et connaissances linguistiques). Il en ressort que dans l'ensemble, l'assimilation spatiale des communautés immigrantes ne se fait pas en référence aux francophones majoritaires, mais plutôt en référence à la minorité anglophone. Une forte hétérogénéité s'observe toutefois entre les communautés et au sein de celles-ci.
\end{abstract}

\footnotetext{
ABSTRACT

Analysis of spatial cohabitation between immigrant communities and Francophones and Anglophones in the Montreal metropolitan area

The Montreal metropolitan area has experienced many successive waves of immigration originating from a range of countries in the $20^{\text {th }}$ and $21^{\text {st }}$ centuries, and is home today to numerous communities of diverse

* World Population Program, International Institute for Applied Systems Analysis. Au moment de rédiger cet article, Guillaume Marois était à l'École d'urbanisme et d'architecture de paysage, Université de Montréal (marois@iiasa.ac.at)

** École d'urbanisme et d'architecture de paysage, Université de Montréal (sebastien.lord@umontreal.ca)

*** Centre Urbanisation Culture Société, Institut national de la recherche scientifique (alain.belanger@ucs. inrs.ca)
} 
origins. While assimilation theory expects immigrants gradually to adopt behaviours comparable to those of native groups in terms of residential location, the dynamics of this process may be different in the Montreal metropolitan area, which has historically been inhabited by large native Francophone and Anglophone communities, each with their own distinct patterns of residential location behaviour. This article aims to measure and analyse the residential cohabitation of people of immigrant origin with these Francophone and Anglophone host communities. The study population is selected from the National Household Survey of 2011 and is composed of the population living in households where the principal livelihood provider was born outside the country or has a parent born outside the country. For each of the fifteen largest immigrant communities we have constructed two regression models with, as dependent variables, the proportion respectively of $3^{\text {rd }}$ generation Francophones and $3^{\text {rd }}$ generation Anglophones living in the same district. This enables us to calculate standardised indexes of interaction according to a series of independent variables such as age, sex, length of residence, education, income and languages spoken. We find that in overall terms the spatial assimilation of immigrant communities takes place with reference to the Anglophone minority rather than the Francophone majority. However there is a high degree of heterogeneity both between and within communities.

\section{INTRODUCTION}

$\mathrm{D}$ ans la plupart des sociétés occidentales, deux grandes tendances démographiques auront des impacts économiques et sociaux importants dans les années à venir : le vieillissement de la population et l'augmentation de la diversité ethnique qui découle des politiques d'immigration (Frey, 2006). Au Canada, plus d'une personne sur cinq est aujourd'hui née à l'étranger (Statistique Canada, 2011). L'immigration se concentrant dans les grandes métropoles, la transformation du portrait démographique de celles-ci est d'autant plus importante du fait qu'il s'agit maintenant de la principale source de croissance démographique et qu'elle est beaucoup plus diversifiée qu'elle ne l'était auparavant (Statistique Canada, 2011; Bohnert, Chagnon et Dion, 2015). Au Québec, $85 \%$ des immigrants s'installent dans la région métropolitaine de Montréal (Institut de la statistique du Québec, 2015). En conséquence des nombreuses vagues successives d'immigration qui ont eu lieu au $20^{\mathrm{e}}$ et $21^{\mathrm{e}}$ siècle et de la diversification des origines des immigrants, le portrait ethnoculturel de Montréal s'est nettement transformé pour devenir très pluriethnique (Germain, Rose et Richard, 2012).

L'objectif de cette étude est de mesurer et d'analyser la cohabitation spatiale des personnes issues de l'immigration avec les communautés francophone et anglophone. Plus précisément, nous cherchons à mesurer l'effet net de l'origine ethnoculturelle sur le choix de la composition linguistique du voisinage. À cette fin, nous avons construit des modèles statistiques permettant d'isoler l'effet des caractéristiques individuelles sur une caractéristique du secteur de résidence (soit la proportion de francophones et d'anglophones). Ces modèles permettent en outre de vérifier si les caractéristiques individuelles généralement associées à l'assimilation spatiale telles que le revenu, l'éducation et la durée de résidence agissent de la même façon d'un groupe ethnoculturel à l'autre.

Bien que l'intégration des immigrants se rapporte à plusieurs aspects, dont la langue et l'emploi, la question du lieu de résidence et des trajectoires résidentielles demeure cruciale (Séguin, Rose et Mongeau, 2003). Plus spécifiquement, les dynamiques spatiales et la ségrégation résidentielle des immigrants intéressent les chercheurs depuis plusieurs décennies. Suivant l'École de 
Chicago, l'intégration résidentielle des immigrants est bien documentée et propose un cadre théorique rigoureux empiriquement validé (Andersen, 2010; Apparicio, Leloup et Rivet, 2006; Fong et Gulia, 1999; Logan, Zhang et Alba, 2002; Massey et Denton, 1985; Park et Burgess, 1925). Selon Andersen (2010), les immigrants tendent à s'établir à leur arrivée dans des secteurs où les loyers sont moins chers et de moins bonne qualité, mais plus près des emplois manufacturiers; puis au fil du temps, ils finissent par adopter des comportements de plus en plus semblables à ceux des natifs et se dirigeraient vers des quartiers périphériques, un processus que l'on nomme assimilation spatiale. Il en résulte ainsi une ségrégation résidentielle relativement importante pour les immigrants récents, souvent économiquement plus précaires, qui tend cependant à diminuer avec les années et lorsque les conditions économiques s'améliorent. Cette tendance peut être altérée lorsque de la discrimination dans le marché immobilier ou des barrières structurelles limitent les opportunités résidentielles pour certains groupes (Charles, 2003). Cette dynamique nommée en anglais «place stratification model » a pour conséquence de maintenir les ségrégations spatiales même pour les groupes socioéconomiques favorisés. Plus récemment, plusieurs chercheurs ont aussi observé une préférence pour vivre avec son groupe ethnique chez certains immigrants des classes moyennes et favorisées. Cette préférence serait le reflet d'un ethnocentrisme naturel plutôt que de contraintes sociales ou d'hostilité entre les groupes (Brown et Chung, 2008; Charles, 2003; Chung et Brown, 2007; Wen et collab. 2009; Walton, 2012). Cette dynamique résidentielle appelée " resurgent ethnicity» en anglais favorise également la ségrégation spatiale sans pour autant amener avec elle des conséquences négatives.

À Montréal, des études ont déjà montré qu'en général, l’isolement résidentiel des immigrants est nettement moindre que dans les autres métropoles nord-américaines (Apparicio, Leloup et Rivet, 2006; Brown et Chung, 2006; Marois et Lord, 2017). La dynamique attendue par la théorie de l'assimilation spatiale s'y observe toutefois plutôt clairement, mais le processus du phénomène s'opère de façon différente entre les communautés. Certaines, à caractéristiques égales, présentent des tendances beaucoup plus fortes à l'isolement résidentiel (Marois et Lord, 2017). Par ailleurs, dans un contexte où plusieurs communautés peuvent coexister dans un même voisinage, une communauté qui n'est pas statistiquement isolée peut néanmoins n'avoir que très peu de contact avec la population d'accueil. Cette question est particulièrement intéressante, car une concentration spatiale de populations vulnérables peut se répercuter en un accès limité aux ressources de la ville, à l'exclusion du marché du travail, en un plus haut taux de décrochage scolaire et en un plus haut risque de stigmatisation (Fitoussi, Laurent et Joël, 2004; Karsten et collab. 2006 ; Sélimanovski, 2008; Shihadeh et Flynn, 1996; van Kempen et şule Özüekren, 1998z). En ce sens, l'étude de la cohabitation des populations immigrantes avec la population native est complémentaire à celle de l'isolement résidentiel et permet d'apporter des éléments nouveaux à l'étude de la dynamique résidentielle et spatiale de l'intégration des immigrants.

Montréal présente par ailleurs une situation unique en Amérique du Nord. La population native est traditionnellement composée des francophones démographiquement majoritaires et des anglophones, minoritaires quantitativement, mais historiquement en position dominante, ce qui lui confère un certain pouvoir d'attraction (Brunet, 1955; Séguin, 1987; Vaillancourt, 1985). Du fait de cette réalité historique et politique, une forte ségrégation spatiale s'observe entre les natifs selon l'appartenance ethnolinguistique (Apparicio et Séguin, 2002), conséquence notamment des préfé- 
rences en matière de portrait linguistique du voisinage (Marois et Bélanger, 2014a ; Marois et Bélanger, 2014b ; Paillé, 2000). Selon le portrait dressé par Apparicio et Séguin (2002), 58 \% des francophones ou des anglophones devraient ainsi déménager pour obtenir une distribution spatiale égale des deux groupes.

Dans un contexte où les politiques d'immigration et d'intégration cherchent à inclure les immigrants à la majorité francophone pour éviter son déclin (Gouvernement du Québec, 2015), la question de la cohabitation spatiale avec ce groupe est cruciale. Bélanger et ses collaborateurs (Bélanger, Lachapelle et Sabourin, 2011; Bélanger, Sabourin et Lachapelle, 2011) ont fait état d'une intégration linguistique déficiente vers le français pour la plupart des communautés allophones non francotropes ${ }^{1}$, tant à la première qu'à la deuxième génération. Bourbeau et ses collaborateurs (2011) montrent quant à eux qu'un lien étroit existe entre la composition linguistique des quartiers et les comportements linguistiques des allophones. En somme, la langue prédominante du milieu de vie est un déterminant parmi d'autres de l'intégration linguistique (Carpentier, 2004).

\section{MÉTHODE ET DONNÉES}

Les indicateurs de ségrégation spatiale peuvent fournir une description géographique détaillée des lieux de résidence des communautés immigrantes, notamment en ce qui a trait à leur isolement ou à leur exposition spatiale à d'autres groupes. Cependant, ils ne peuvent mesurer l'effet des déterminants individuels sur ces dynamiques spatiales, pas plus qu'ils ne tiennent compte des effets de composition des communautés qui peuvent agir sur la localisation résidentielle. L'approche utilisée dans cette étude est basée sur les modèles « locational attainment " (Alba et Logan, 1992) dans lesquels la variable dépendante est une caractéristique du quartier de résidence, alors que les variables indépendantes sont des caractéristiques individuelles.

Plusieurs études utilisent le revenu médian du quartier ou la proportion de «Blancs » comme variable d'analyse pour vérifier la théorie de l'assimilation spatiale pour certains groupes. En somme, elles cherchent à déterminer si un groupe minoritaire ou vulnérable tel que les « Noirs » ou les «Hispaniques » a le même accès à un quartier désirable qu'un "Blanc » ayant les mêmes caractéristiques (Cort, 2011; Glikman et Semyonov, 2012; Logan et collab. 1996; Myles et Hou, 2003). Étudiant le cas spécifique de Montréal, Marois et Lord (2017) ont construit des modèles similaires en utilisant comme variable dépendante la proportion de la population du quartier appartenant au même groupe, permettant ainsi d'effectuer un contrôle statistique sur la propension à l'isolement résidentiel. Les conclusions de l'étude montrent que la théorie de l'assimilation spatiale n'opère pas de la même façon chez toutes les communautés. En somme, même si aucune communauté immigrante n'est fortement isolée, certains sous-groupes présentant une combinaison de caractéristiques particulières le sont nettement plus.

Suivant cette approche, pour cette étude, nous avons construit deux modèles ayant comme variable dépendante respectivement la proportion de la population du quartier de résidence

1. Les communautés allophones non francotropes sont celles provenant de pays n'ayant pas de lien important avec la francophonie ou la langue française. 
appartenant à la communauté francophone et celle appartenant à la communauté anglophone. Pour une communauté immigrante $c$, les modèles peuvent alors s'exprimer par l'équation 1 :

(1) $Y^{c}=a^{c}+\beta_{1}^{c} X_{i 1}^{c}+\beta_{2}^{c} X_{i 2}^{c} \ldots+e_{i}^{c}$

où

$\mathrm{Y}^{c}$ est la proportion de la population du quartier appartenant au groupe francophone ou anglophone;

$\mathrm{X}^{\mathrm{c}}{ }_{\mathrm{ik}}$ sont un ensemble de $\mathrm{k}$ caractéristiques pour un individu i de la communauté $\mathrm{c}$;

$\beta_{k}^{c}$ sont les paramètres linéaires des caractéristiques $\mathrm{k}$ pour la communauté $\mathrm{c}$.

Un intérêt particulier de ce modèle est que la valeur de l'ordonnée à l'origine $\alpha^{c}$ correspond exactement à l'indice d'interaction résidentielle (Apparicio, 200o; Bell, 1954) lorsqu'il n'y a pas de variables indépendantes. Pour une communauté cible, cet indice correspond à la proportion moyenne de la population appartenant à un groupe de référence (soit, dans notre cas, la proportion de francophones ou d'anglophones) et varie de o à 1 . Il mesure ainsi l'interaction spatiale du groupe cible avec les personnes du groupe de référence, c'est-à-dire la probabilité pour une personne d'une communauté $\mathrm{c}$ de rencontrer un membre du groupe de référence b dans son quartier. Pour une communauté de référence $c$ et un groupe cible $b$, l'indice d'interaction $\mathrm{I}_{\mathrm{b}}{ }^{\mathrm{c}}$ peut s'écrire par l'équation 2.

$$
\text { (2) } I_{b}^{c}=\sum_{j=1}^{n}\left[c_{j} / C\right]\left[b_{j} / t_{j}\right]
$$

où

$c_{j}$ est la population du groupe cible (communauté c) dans le secteur $j$;

$b_{i}$ est la population du groupe de référence (francophones ou anglophones) dans le secteur $j$;

C est la population de la communauté c dans l'ensemble de la région;

$t_{i}$ est la population totale du secteur $j$.

Un indice d'interaction avec les francophones de o indiquerait ainsi qu'aucun membre de la communauté $\mathrm{c}$ ne vit dans un secteur où résident des francophones. S'il y a une distribution spatiale égale de la population, les indices d'interaction avec les francophones et les anglophones devraient correspondre respectivement aux proportions de francophones et d'anglophones dans l'ensemble du territoire. Comme ces proportions sont nettement différentes, les indices bruts ne sont pas comparables et nécessitent une modification pour pouvoir mesurer la sous-exposition ou la surexposition des communautés à chacun de ces groupes de référence. L'indice d'interaction doit alors être transformé pour obtenir un indice d'interaction modifié ${ }_{c} \mathrm{IM}_{\mathrm{b}}$ (Apparicio, 2000 ; Bell, 1954) qui prend en compte la proportion relative des groupes d'interaction en centrant l'indice sur 1. Cet indice modifié s'exprime par l'équation 3.

$$
\text { (3) } I M_{b}^{c}=\frac{I_{b}^{c}}{B / T}
$$

où

B est la population du groupe de référence (francophones ou anglophones) dans la région métropolitaine;

T est la population totale de la région métropolitaine. 
$\mathrm{Un} \mathrm{IM}_{\mathrm{b}}{ }^{\mathrm{c}}$ de 1 indiquerait donc que la proportion moyenne de personnes du groupe de référence (francophones ou anglophones) dans les quartiers où vivent les membres de la communauté c est la même que la proportion observée dans l'ensemble de la région métropolitaine. Un indice supérieur à 1 indiquerait quant à lui une surexposition de la communauté c au groupe de référence, alors qu'un indice inférieur à 1 indiquerait une sous-exposition.

En revenant à l'équation 1 , les paramètres $\beta$ indiquent alors l'impact des variables individuelles sur l'indice d'interaction. En d'autres mots, l'analyse des $\beta$ nous permet de mesurer comment les caractéristiques individuelles influencent la propension à vivre avec le groupe de référence. Lorsqu'il y a un grand nombre de caractéristiques intégrées, le modèle effectue un contrôle statistique sur $\mathrm{I}_{\mathrm{b}}{ }^{\mathrm{c}}$ (et par déduction, sur $\mathrm{IM}_{\mathrm{b}}{ }^{\mathrm{c}}$ ). Les $\alpha^{\mathrm{c}}$ peuvent alors être assimilés à des indices d'interaction standardisés $\mathrm{IS}_{\mathrm{b}}{ }^{\mathrm{c}}$ (voir équation 4), soit les indices correspondant à un profil de référence défini par les catégories omises des variables indépendantes.

$$
\text { (4) } I S_{b}^{c}=\alpha^{c}
$$

où

$\alpha^{c}$ est la valeur de l'ordonnée à l'origine de l'équation 1.

Tel que décrit à l'équation 5 , en effectuant la même transformation à $\mathrm{IS}_{\mathrm{b}}{ }^{\mathrm{c}}$ que celle effectuée pour obtenir $\mathrm{IM}_{\mathrm{b}}{ }^{\mathrm{c}}$, nous obtenons un indice d'interaction modifié et standardisé $\mathrm{IMS}_{\mathrm{b}}{ }^{\mathrm{c}}$, c'est-àdire qui prend en compte la taille des populations de référence et la composition de la population du groupe cible.

$$
\text { (5) } I M S_{b}^{c}=\frac{I S_{b}^{c}}{B / T}
$$

Les modèles locational attainment peuvent générer des problèmes dus à l'autocorrélation spatiale, puisque les individus vivant dans un même secteur ont tous la même valeur pour la variable dépendante, celle-ci étant en partie influencée par leur présence dans le secteur (Cort, 2011; Logan et collab. 1996; Pelletier, 2012). Ceci a pour effet de sous-estimer l'erreur-type des paramètres, puisque les erreurs sont corrélées. Dans une telle situation, les auteurs utilisant ce type de modèle recommandent généralement d'estimer les paramètres par la méthode des moindres carrés généralisés faisables (feasible generalized least squares) (Cort, 2011; Myles et Hou, 2003), ce qui permet de prendre en compte la corrélation des termes d'erreur à l'intérieur d'un même secteur (Greene, 1997).

Nos analyses nécessitent une base de données individuelles qui contient une information sur la localisation résidentielle à un petit niveau géographique en plus des caractéristiques individuelles, dont la dimension ethnoculturelle. La base de données la plus récente fournissant ce genre d'information est l'Enquête nationale auprès des ménages de 2011 (ENM) (Statistique Canada, 2013), qui contient non seulement les caractéristiques individuelles telles que le sexe, l'âge, la structure familiale, le revenu, l'éducation, l'année d'immigration, la langue, etc., mais aussi des informations sur le lieu de naissance du répondant et de ses parents qui permettent de construire des communautés immigrantes basées sur le pays d'origine de la première et de la deuxième génération d'immigrants. L'ENM découpe aussi la région métropolitaine de Montréal en 921 secteurs de recensements qui comptent entre 2500 et 8000 habitants et peuvent être assimilés à des quartiers. 
Cependant, puisque l'ENM, qui remplace le formulaire long des recensements précédents, n'est pas obligatoire, le taux de réponse est de $70 \%$ et peut varier fortement entre les groupes. En conséquence, il est possible que le biais de non-réponse soit plus grand pour certains groupes spécifiques tels que les immigrants récents ou ceux ne parlant ni français ni anglais, ce qui aurait pour effet de surestimer l'interaction avec les groupes de références.

Nous considérons comme appartenant à une communauté immigrante les individus vivant dans un ménage où le principal soutien est soit né à l'étranger, soit a un parent né à l'étranger², en excluant ceux venus de France, de Grande-Bretagne ou des États-Unis pour des raisons historiques. La population immigrante de Montréal est très hétérogène par ses origines et il ne serait pas possible d'analyser toutes les communautés dans leur diversité. Nous avons regroupé les pays qui ont des similarités contextuelles ou géographiques et avons conservé les 15 plus importantes communautés. Conformément à la méthode habituelle pour ce genre d'analyse, les caractéristiques sont donc celles du principal soutien (Myles et Hou, 2003; Rosenbaum et Friedman, 2001), de manière à avoir des informations plus précises sur la localisation résidentielle de la deuxième génération. Dans le cas contraire, les comportements observés seraient biaisés par ceux des enfants vivant avec leurs parents (première génération). La population des groupes de références, soit les francophones et anglophones, est quant à elle formée des personnes vivant dans un ménage dont le principal soutien est de $3^{\mathrm{e}}$ génération ou plus et a comme langue maternelle le français ou l'anglais. Il s'agit en somme d'une population qui, pour l'essentiel, correspond aux descendants des colons français et britanniques et des populations immigrantes anciennes dont les descendants se sont assimilés à l'un ou l'autre de ces groupes.

\section{ANALYSE DESCRIPTIVE DES COMMUNAUTÉS IMMIGRANTES}

Les 15 communautés immigrantes de la région métropolitaine de Montréal construites dans le cadre de cet article sont les suivantes : les Italiens, les Maghrébins, les Haïtiens, les Libanais, les Grecs, les Chinois, les Vietnamiens, les Portugais, les Roumains, les Philippins, les Sud-Américains, les Centre-Américains, les Subsahariens, les Arabes (autres) et les Sud-Asiatiques. L'effectif total de ces communautés, qui comptent toutes au moins 30 ooo membres, représente 1,1 million de personnes, soit $30 \%$ de la région métropolitaine (3,8 millions). La plus grande est la communauté italienne (167 000), suivie des Maghrébins (140 000) et des Haïtiens (117 000).

Seules les communautés haïtienne, centre-américaine et philippine ont une proportion similaire d'hommes et de femmes comme principal soutien du ménage. Les hommes sont prédominants chez tous les autres groupes, particulièrement chez les Sud-Asiatiques, Maghrébins, Libanais et ceux des autres pays arabes. Le faible rapport de masculinité des Philippins peut être causé par le fait que plusieurs immigrants de ce groupe sont arrivés par le programme des Aides familiaux résidants qui recrute surtout des femmes. La prédominance des femmes comme principal soutien chez les Centre-Américains et les Haïtiens peut être mise en relation avec leur forte proportion de

2. Selon cette définition, les personnes de $1^{\text {ère }}$ et $2^{\mathrm{e}}$ génération qui vivent dans un ménage dont le principal soutien ne remplit pas ces conditions ne sont pas considérées comme faisant partie d'une communauté immigrante (par exemple, les enfants adoptés ou le conjoint immigrant d'un couple mixte). 
familles monoparentales $(25,1 \%$ et $29,5 \%$ respectivement, alors que les proportions varient de $6,2 \%$ à $16,9 \%$ pour les autres communautés). À l'opposé, les familles avec enfants sont très nombreuses chez les Sud-Asiatiques (75,1\%), les Maghrébins (72,8 \%) et les Libanais (69,5\%).

Les communautés italienne et grecque sont essentiellement issues d'anciennes vagues d'immigration. Respectivement, $90 \%$ et $80 \%$ d'entre eux ont immigré avant 1971 ou sont de $2^{\mathrm{e}}$ génération. À l'inverse, une forte proportion des Maghrébins, Chinois, Roumains et Subsahariens sont des immigrants récents : plus de $40 \%$ d'entre eux sont arrivés dans les années 2000 et très peu sont nés au Canada. Concernant les Haïtiens et les Vietnamiens, la plupart d'entre eux sont arrivés entre 1971 et 1990, à la suite des troubles politiques dans leurs pays d'origine. Ces périodes d'arrivée fort différentes d'un groupe à l'autre influencent la structure par âge des principaux soutiens des ménages. Le quart des Italiens et des Grecs vivent dans un ménage mené par une personne de 65 ans et plus, ce qui en fait les plus âgés. Cette proportion chez les Maghrébins et les Subsahariens, qui sont souvent d'immigration récente, est inférieure à $5 \%$.

Le capital humain tel que mesuré par le niveau d'éducation et la connaissance des langues officielles diffère fortement entre les communautés immigrantes. Avec des proportions d'environ $50 \%$ de personnes vivant dans des ménages où le principal soutien détient au minimum un baccalauréat, les Roumains, les Chinois, les Maghrébins et les Arabes (autres) sont les plus éduqués. Tel que mentionné précédemment, ces personnes sont souvent d'immigration récente, ce qui suggère qu'ils ont pour la plupart immigré par le programme des travailleurs qualifiés. Les vagues d'immigration plus anciennes sont généralement moins éduquées, étant arrivées avant l'instauration des programmes d'immigration économique et ayant eu moins accès à l'éducation supérieure que les cohortes plus récentes. Beaucoup de Portugais, Grecs et Italiens vivent ainsi dans un ménage où le principal soutien n'a aucun diplôme $(38,0 \%, 31,7 \%$ et $25,1 \%$ respectivement) et très peu ont un diplôme universitaire $(11,8 \%, 18,9 \%$ et $18,7 \%)$. Parmi les communautés dont plusieurs ont immigré comme réfugiés, de fortes proportions n’ont aussi aucun diplôme, tel que les Vietnamiens (25\%), les Centre-Américains (22,8 \%), les Sud-Asiatiques (20,6 \%) et les Haïtiens (18,6 \%).

De fortes proportions de personnes vivant dans un ménage dont le principal soutien connaît à la fois le français et l'anglais s'observent chez plusieurs communautés, particulièrement chez les Italiens (81,3\%), les Roumains (77,6 \%) et les Libanais (76,6 \%). Chez d'autres communautés, c'est la connaissance seule de l'anglais qui domine, tel que chez les Philippins ( $76,7 \%)$, les SudAsiatiques (66,6\%) et les Chinois (52,9\%). Une seule communauté compte une proportion de locuteurs de l'anglais inférieure à $50 \%$, les Haïtiens, dont $53 \%$ d'entre eux ne connaissent en effet que le français. Mentionnons finalement que chez les Chinois et les Vietnamiens, une proportion appréciable ne connaît ni le français ni l'anglais (respectivement 15,6 \% et $11 \%$ ). Ces proportions sont marginales chez les autres communautés étudiées.

Puisque les communautés immigrantes ont des compositions très différentes en termes de capital humain, statut générationnel et durée d'arrivée, il n'est pas étonnant que la distribution de leurs membres selon la classe de revenu soit également très variée. Des proportions très élevées de personnes vivant dans un ménage dont le revenu se trouve dans le quintile le plus pauvre s'observent chez les Maghrébins (37,7 \%), les Subsahariens (37,2 \%), les Sud-Asiatiques (35,8 \%) et les Centre-Américains $(34,6 \%)$. Les communautés avec les plus fortes proportions de personnes se trouvant dans le quintile le plus riche sont les Italiens $(22,6 \%)$, ceux des autres pays arabes 
$(19,8 \%)$ et les Roumains (19,5\%). Les Italiens forment en somme le seul groupe à être globalement plus riche que la moyenne de la région métropolitaine de Montréal.

En bref, les communautés immigrantes sont très différentes les unes des autres en ce qui a trait à leur composition socioéconomique et à leurs parcours migratoires. Cette description des groupes étudiés tend ainsi à soutenir l'approche statistique multivariée pour mesurer la propension nette des communautés à vivre avec les francophones ou les anglophones, car l'observation des indicateurs bruts d'interaction résidentielle peut contenir un effet de composition biaisant la comparaison des comportements entre les communautés.

\section{ANALYSE STATISTIQUE DE L'INTERACTION RÉSIDENTIELLE}

\section{L’interaction résidentielle avec les francophones et anglophones}

À la figure 1, nous présentons en premier lieu les indices d'interaction brut $\mathrm{I}_{\mathrm{b}}{ }^{\mathrm{c}}$ (plein) et standardisé $\mathrm{IS}_{\mathrm{b}}{ }^{\mathrm{c}}$ (hachuré) avec les francophones (en bleu) et les anglophones (en rouge) pour les 15 communautés immigrantes étudiées. Tel que mentionné dans la partie méthodologique, les indices standardisés correspondent aux ordonnées à l'origine des modèles de régression et correspondent donc à un profil type 3 .

FIGURE 1

Indice d’interaction (brut et standardisé) avec les francophones et anglophones, RMR de Montréal, 2011

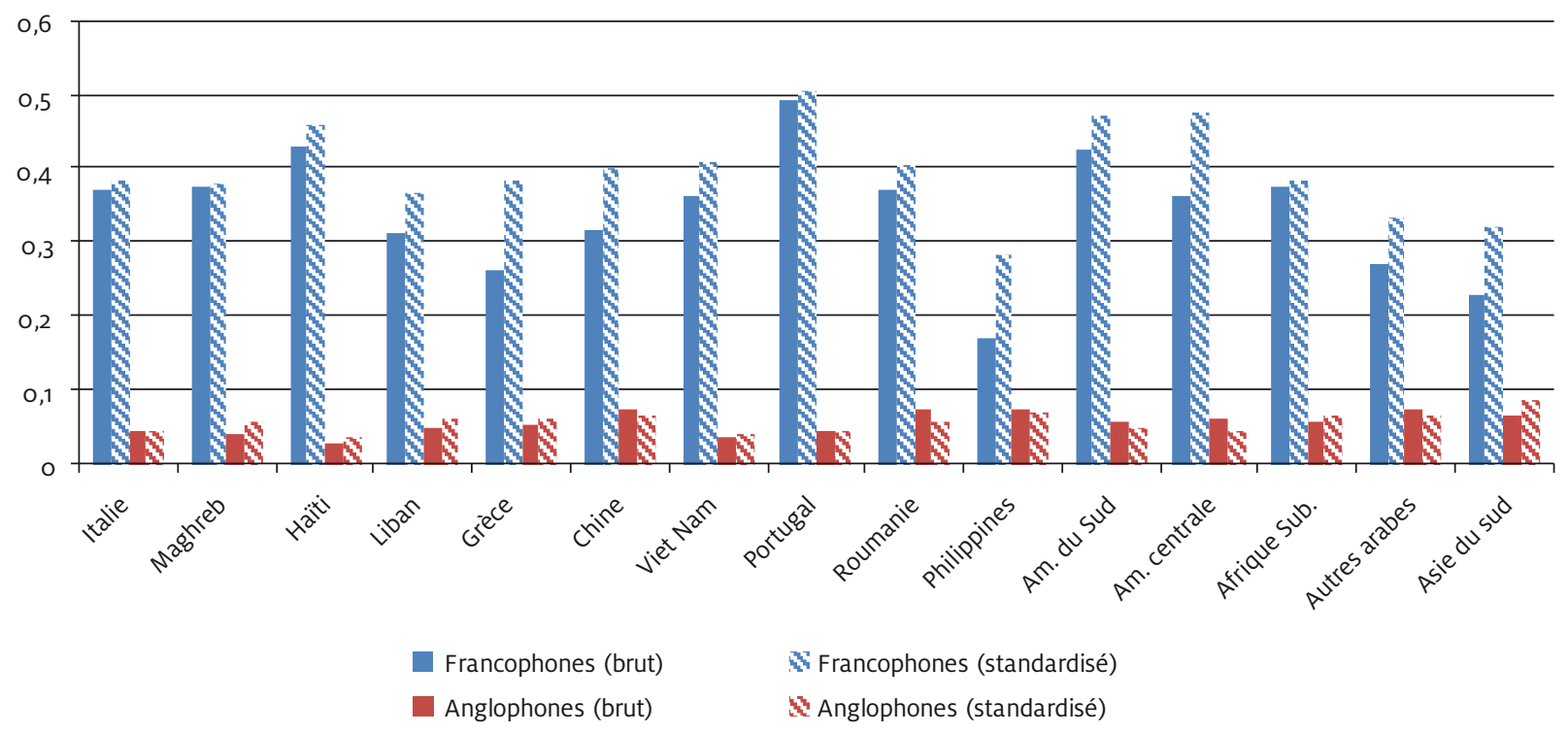

3. Le profil type correspond aux catégories omises des modèles, c'est-à-dire une personne vivant dans un ménage dont le principal soutien est un homme âgé entre 30 et 34 ans détenteur d'un baccalauréat, qui connaît le français et l'anglais, qui a un revenu situé dans le ze quintile, est en couple sans enfant et est un immigrant de première génération arrivé entre 1981 et 1990. 
En premier lieu, nous notons que pour toutes les communautés, les indices d'interaction avec les francophones sont plus grands que les indices d'interaction avec les anglophones, ce qui est normal puisque les francophones tels que définis dans cette étude forment 54,5\% de la population de la région métropolitaine contre 5,1\% pour les anglophones. L'indice brut d'interaction avec les francophones le plus faible s'observe chez les Philippins et atteint 0,169, signifiant qu'en moyenne, l'on trouve $16,9 \%$ de francophones dans les quartiers où vivent les Philippins. À l'opposé, l'indice brut le plus fort s'observe chez les Portugais et atteint 0,491. Globalement, les indices standardisés sont plus élevés que les bruts, avec toutefois des variations importantes entre les communautés. Pour l'interaction avec les anglophones, les indices bruts varient de 0,025 (Haïtiens) à 0,075 (Philippins). Tel que mentionné précédemment, ces indices non ajustés ne renseignent pas sur la sur- ou sous-exposition aux francophones ou anglophones, mais permettent néanmoins de voir qu'aucune communauté immigrante n'est complètement isolée de l'un ou l'autre des groupes de références.

La figure 2 illustre les indices modifiés $\mathrm{IM}_{\mathrm{b}}{ }^{\mathrm{c}}$ et les indices modifiés et standardisés $\mathrm{IMS}_{\mathrm{b}}{ }^{\mathrm{c}}$. Ces indices prennent en compte la taille des groupes de référence et permettent ainsi de mesurer la surexposition ou la sous-exposition des communautés immigrantes aux francophones et anglophones. Un premier constat est que toutes les communautés immigrantes sont sous-exposées aux francophones, toutefois à des degrés différents. En règle générale, les communautés francotropes (Haïtiens, Portuguais, Sud-Américains, Maghrébins, etc.) sont un peu moins sous-exposées que les autres communautés, mais le degré de sous-exposition demeure quand même élevé. De fait, seule la communauté portugaise a un indice se rapprochant de 1 (qui indiquerait l'absence de sous-exposition). Les communautés philippine, sud-asiatique, grecque et chinoise sont les plus sous-exposées avec des $\mathrm{IM}_{\mathrm{b}}{ }^{\mathrm{c}}$ inférieurs à o,6. Atteignant un plancher de o,311, l'indice de la communauté philippine se démarque de tous les autres par son bas niveau. Il indique en somme que les personnes de cette communauté vivent dans des quartiers où il y a environ 3 fois moins de francophones que ce qui serait attendu s'il y avait une distribution spatiale égale de la population. Pour toutes les communautés, les indices modifiés et standardisés $\left(\mathrm{IMS}_{\mathrm{b}}{ }^{\mathrm{c}}\right)$ tendent à réduire la sousexposition observée par l'IM ${ }_{b}^{c}$, mais la sous-exposition demeure généralement très importante. Cela signifie que la composition des communautés n'explique que partiellement la sous-exposition au groupe francophone.

Concernant l'interaction avec les anglophones, la situation est nettement différente. D'abord, très peu de communautés immigrantes sont sous-exposées à la communauté anglophone. Seule la communauté haïtienne a un $\mathrm{IM}_{\mathrm{b}}{ }^{\mathrm{c}}$ plutôt bas $(0,502)$ et dans une moindre mesure, la communauté vietnamienne $(0,717)$. Les autres communautés ont soit des indices se rapprochant de la norme de 1 indiquant l'absence de sur- ou sous-exposition, soit des indices nettement supérieurs à 1. Parmi celles-ci, l'on retrouve notamment plusieurs communautés non francotropes, dont les Chinois $(1,411)$, les Philippins $(1,466)$ et les Sud-Asiatiques $(1,244)$. La communauté roumaine, qu'on classe généralement dans la catégorie francotrope étant donné sa langue d'origine latine, se démarque aussi des autres communautés francotropes par une très forte surexposition aux anglophones $(1,415)$. Les indices $\mathrm{IMS}_{\mathrm{b}}{ }^{\mathrm{c}}$ révèlent quant à eux la même dynamique, avec quelques différences d'amplitude, notamment pour la communauté sud-asiatique pour laquelle l'IMS $_{\mathrm{b}}{ }^{\mathrm{c}}$ est nettement plus élevé que l'IM ${ }_{b}{ }^{c}(1,668$ contre 1,244$)$. 


\section{FIGURE 2}

Indice d'interaction modifié (brut et standardisé) avec les francophones et anglophones,

RMR de Montréal, 2011

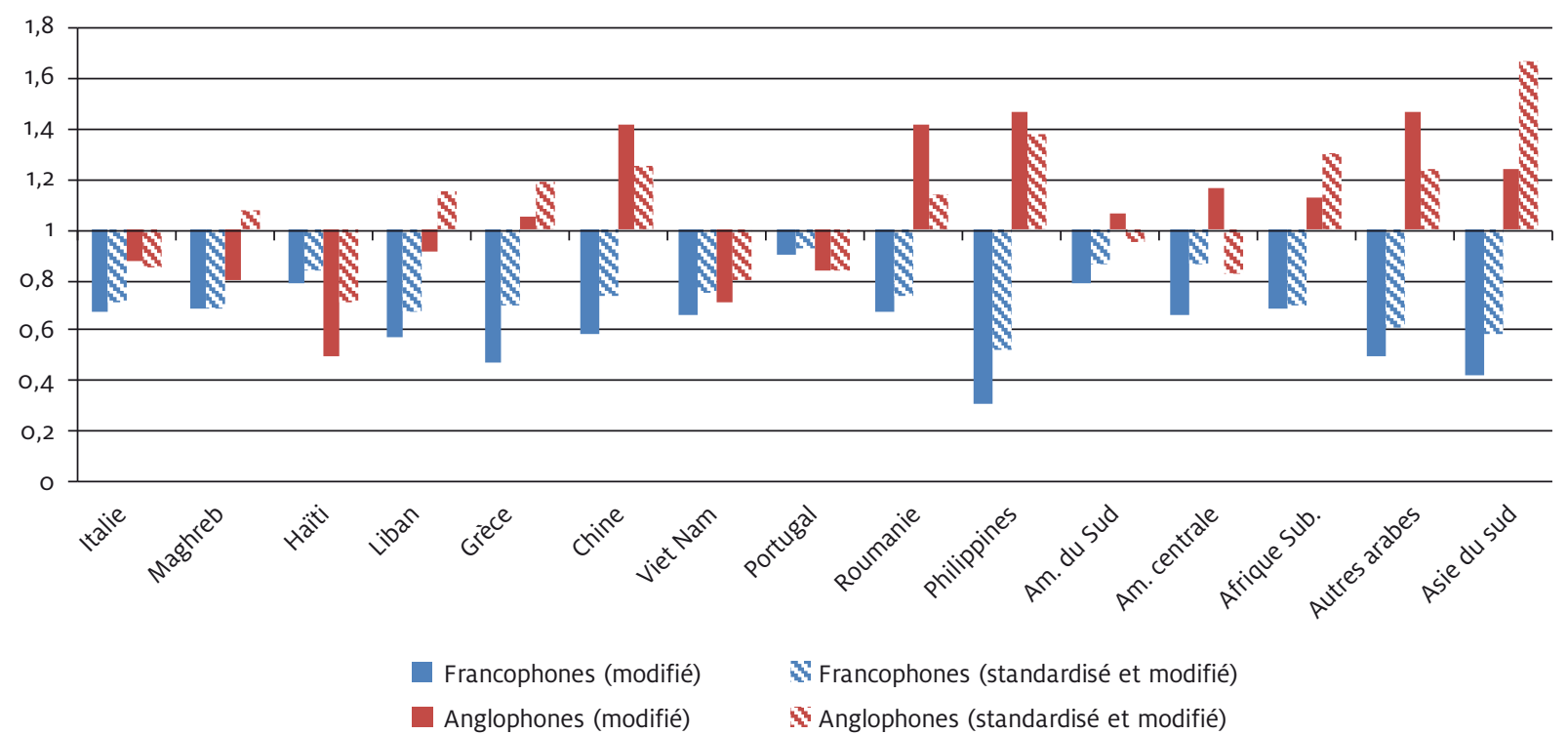

En somme, l'analyse des indices d'interaction aux communautés anglophones révèle la dynamique générale suivante : les communautés non francotropes tendent à vivre dans des quartiers à forte présence anglophone alors que les communautés francotropes sont soit sous-exposées aux deux communautés d'accueil, ce qui indiquerait alors un certain isolement résidentiel, soit sousexposées aux francophones et exposées normalement aux anglophones. Soulignons finalement que seules les communautés portugaise et, dans une moindre mesure, sud-américaine semblent occuper le territoire de manière plutôt homogène. Cela confirme par ailleurs une précédente étude dans laquelle ces communautés se démarquaient par leur très faible degré de ségrégation résidentielle (Marois et Lord, 2017). En considérant les indices standardisés, nous pouvons rajouter les Centre-américains. Bien que leur $\mathrm{IM}_{\mathrm{b}}{ }^{\mathrm{c}}$ soit faible $(0,666)$, leur $\mathrm{IMS}_{\mathrm{b}}{ }^{\mathrm{c}}$ se rapproche quant à lui de $1(0,868)$, signifiant que leur sous-exposition observée aux francophones s'explique en grande partie par leur composition socioéconomique.

\section{Les déterminants socioéconomiques de l’interaction résidentielle aux francophones et anglophones}

Tel que nous l'avons vu aux figures 1 et 2, la composition socioéconomique des communautés immigrantes a parfois une influence importante sur leur interaction résidentielle. La section qui suit présente les principaux paramètres des régressions permettant de saisir l'effet des caractéristiques individuelles sur les indices d'interaction. Comme chaque communauté a un modèle spécifique, les analyses permettent de mesurer les effets parfois divergents entre les communautés d'une même variable. Les paramètres détaillés sont présentés en annexes 1 et 2. 
L'âge du principal soutien a un effet important sur l'interaction résidentielle pour plusieurs communautés, mais va parfois en directions opposées selon le groupe de référence francophone ou anglophone. Pour l'indice d'interaction avec les francophones, la tendance générale chez les communautés italienne, grecque, sud-américaine et centre-américaine est une relation négative indiquant que les plus vieux sont généralement moins exposés que les plus jeunes. Pour l'interaction avec les anglophones, l'effet est généralement contraire. La tendance est particulièrement marquée chez les communautés italienne, grecque, sud-américaine et centre-américaine, mais aussi chez les Chinois et Subsahariens. Cependant, comme ces données concernent plusieurs cohortes, cet effet pourrait aussi résulter d'un changement de comportement chez les cohortes plus jeunes. Cette possibilité est particulièrement vraie pour les communautés issues de vagues plus anciennes d'immigration qui se sont installées avant la prise en charge par le gouvernement du Québec des programmes d'immigration économique accordant une place plus importante à la connaissance du français et avant l'instauration des politiques linguistiques telles que la Charte de la langue française adoptée en 1977.

La durée de résidence est une variable fondamentale de la localisation résidentielle et de la théorie de l'assimilation spatiale (Massey and Denton, 1985). Selon celle-ci, il est attendu que les immigrants vivent une plus grande ségrégation résidentielle à leur arrivée, puis au fil de leur intégration, adoptent des comportements résidentiels semblables aux natifs. De ce fait, la relation entre la durée de résidence et l'interaction résidentielle avec les francophones et anglophones devrait être positive, de même que les paramètres associés à la deuxième génération. Dans les modèles de régression, cette relation est effectivement observée dans certains cas, notamment pour l'interaction avec les anglophones chez les Maghrébins et les Sud-Asiatiques. Pour la deuxième génération de deux parents nés à l'étranger, comme le montre la figure 3, nous observons aussi des paramètres positifs (par rapport à la catégorie de référence qui sont les immigrants arrivés entre 1981 et 1990) et généralement statistiquement significatifs pour l'interaction résidentielle avec les anglophones pour toutes les communautés immigrantes, notamment chez les communautés maghrébine et roumaine pourtant francotropes. Cependant, pour l'interaction avec les francophones, la relation positive attendue pour la deuxième génération ne s'observe que chez les Vietnamiens, Philippins, Subsahariens et ressortissants des autres pays arabes. Les paramètres sont en retour négatifs et fortement significatifs chez les Chinois, les Maghrébins et les Roumains. Cependant, le fait d'être issu d'un couple mixte favorise largement l'interaction résidentielle avec les francophones. Les paramètres pour la $2^{\mathrm{e}}$ génération dont un seul parent est né à l'étranger sont en effet nettement positifs, à l'exception de celui pour la communauté roumaine qui n'est pas significatif, et sont tous supérieurs à ceux dont les deux parents sont nés à l'étranger. Celui associé à la communauté philippine atteint un niveau particulièrement élevé $(0,260)$, ce qui vient complètement contrebalancer la forte propension à la sous-exposition aux francophones observée pour le profil de référence. 


\section{FIGURE 3}

Effet du statut de génération sur l'indice d'interaction avec les francophones et anglophones, RMR de Montréal, 2011

Catégorie de référence: immigrants arrivés entre 1981 et 1990 $x=$ paramètre non significatif $(p>0,05)$

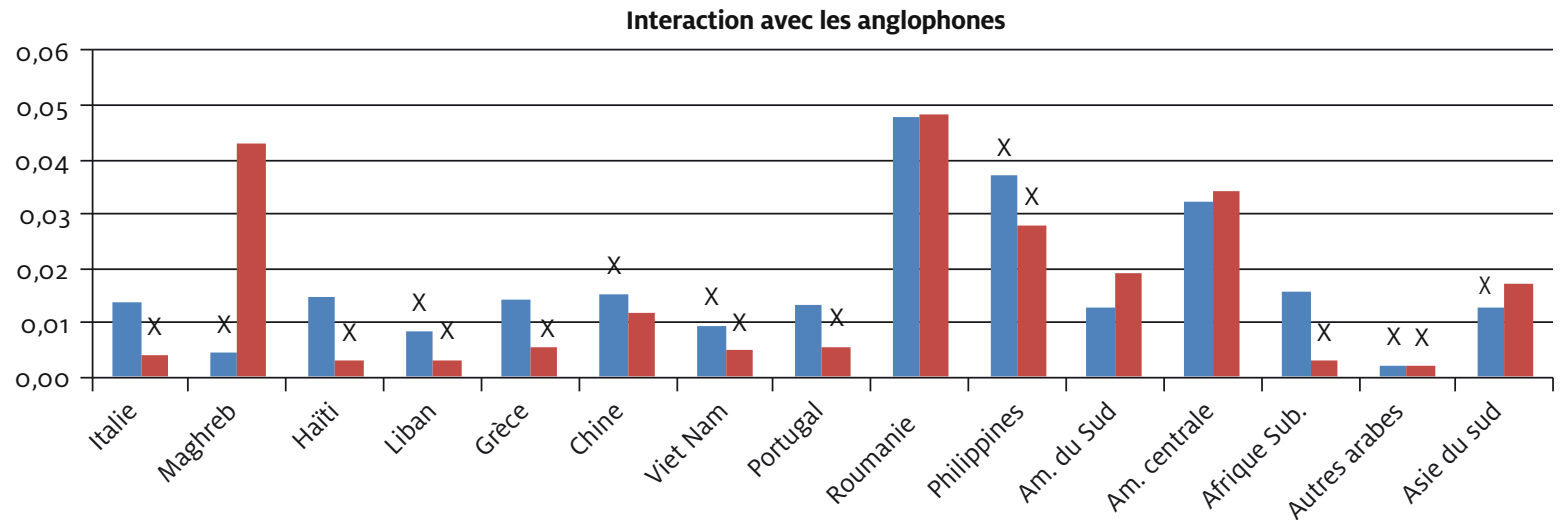

En somme, ces résultats tendent à montrer que l'assimilation spatiale des communautés immigrantes ne se fait pas en référence aux francophones majoritaires, mais plutôt en référence à la minorité anglophone, et ce, même chez les communautés francotropes, sauf pour les couples mixtes où un des parents est natif et l'autre immigrant. Cependant, les paramètres des modèles ne vont pas tous en ce sens. Par exemple, la relation parait inversée pour l'interaction avec les francophones chez les Maghrébins, où il semble y avoir une fracture entre ceux arrivés après 1991 et ceux arrivés avant, ces derniers étant en 2011 nettement moins exposés aux francophones que leurs compatriotes plus récemment arrivés. Peu de différences s'observent au sein des différentes vagues arrivées après 1991. Encore une fois, comme pour l'âge, il pourrait cependant s'agir d'un effet de cohorte.

L'effet attendu pour la variable de la durée de résidence pourrait par contre être absorbé par la variable du revenu qui y est généralement corrélée. La figure 4 montre l'effet du quintile de revenu sur les indices d'interaction résidentielle avec les francophones et anglophones. En règle générale, peu importe la communauté, il appert que les plus pauvres ont moins tendance à être exposés aux francophones. Le paramètre est particulièrement fortement négatif chez les Maghrébins du premier quintile $(-0,08)$. Cependant, la relation n'est pas graduelle. Pour la plupart des commu- 
nautés, le quintile de revenu le plus exposé aux francophones n'est pas le plus riche, mais plutôt le $4^{\mathrm{e}}$. Concernant l'exposition aux anglophones, comme pour l'exposition aux francophones, la relation entre le revenu ne suit pas une tendance linéaire (sauf pour les Sud-Asiatiques). L'on remarque pour la plupart des communautés une nette distinction entre le quintile le plus riche et les autres, montrant que les communautés immigrantes les plus fortunées ont beaucoup plus tendance à vivre dans des quartiers anglophones que leurs compatriotes des classes moyenne et pauvre pour lesquelles les paramètres sont semblables. Cette observation vaut également pour les communautés francotropes comme les Maghrébins, les Libanais et les Sud-Américains.

Une autre variable associée au capital humain est la scolarité. Les régressions montrent que cette dernière agit clairement sur l'exposition résidentielle aux anglophones. Tel qu'illustré à la figure 5, pour toutes les communautés, une tendance claire s'observe entre le plus haut diplôme du chef du ménage et la propension à vivre dans un secteur anglophone. L'ampleur de l'effet diverge toutefois d'une communauté immigrante à l'autre. Avec des coefficients fortement négatifs associés à la catégorie «sans diplôme » (aux alentours de -0,03) par rapport à ceux qui ont un

\section{FIGURE 4}

Effet du quintile de revenu sur l'indice d'interaction avec les francophones et anglophones, RMR de Montréal, 2011

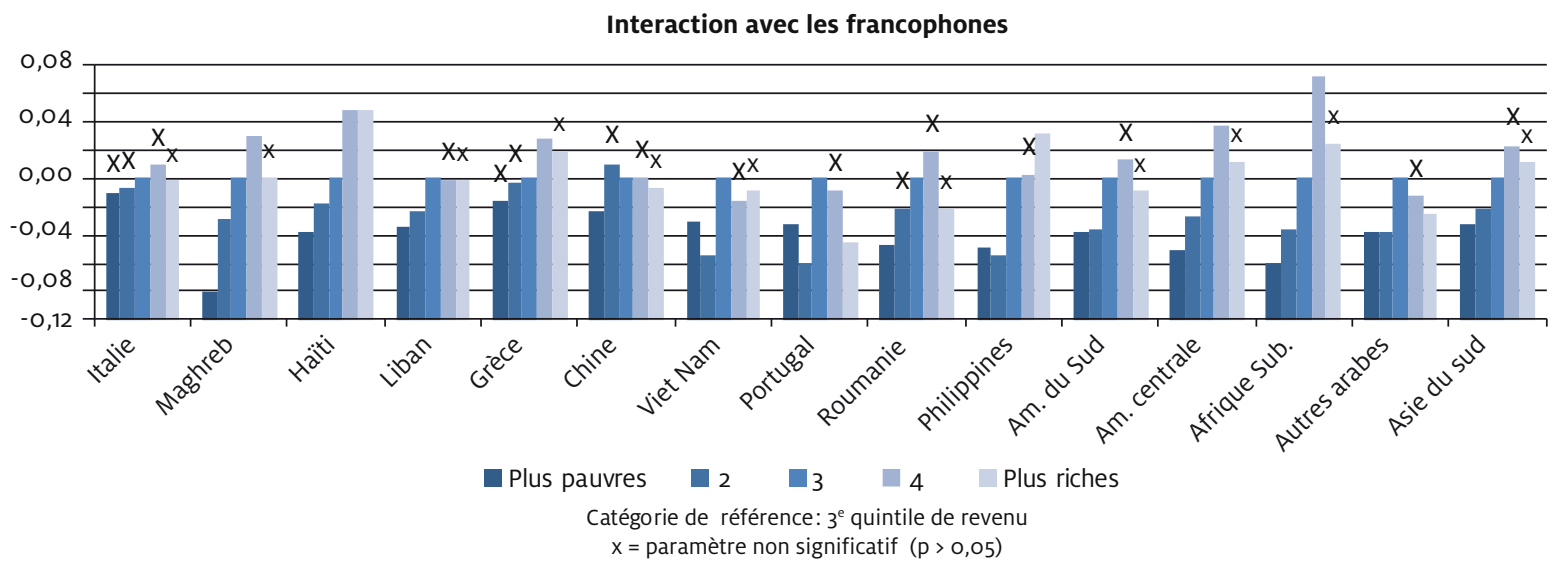

Interaction avec les les anglophones

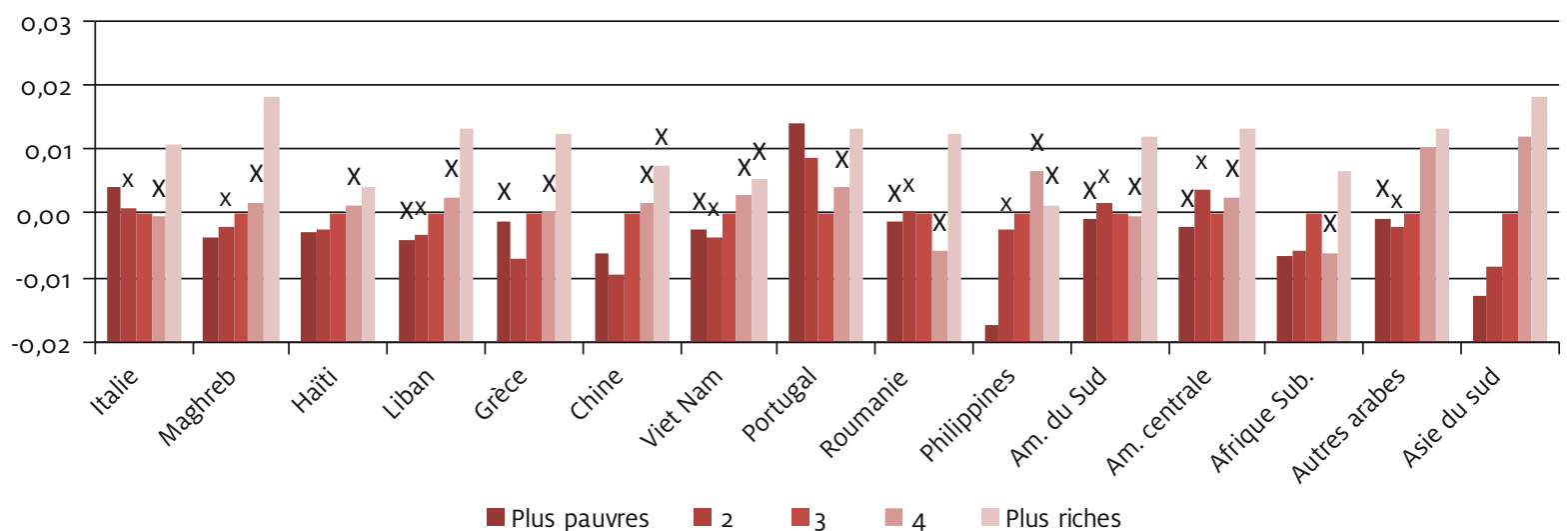


FIGURE 5

Effet de la scolarité sur l'indice d'interaction avec les francophones et anglophones,

RMR de Montréal, 2011

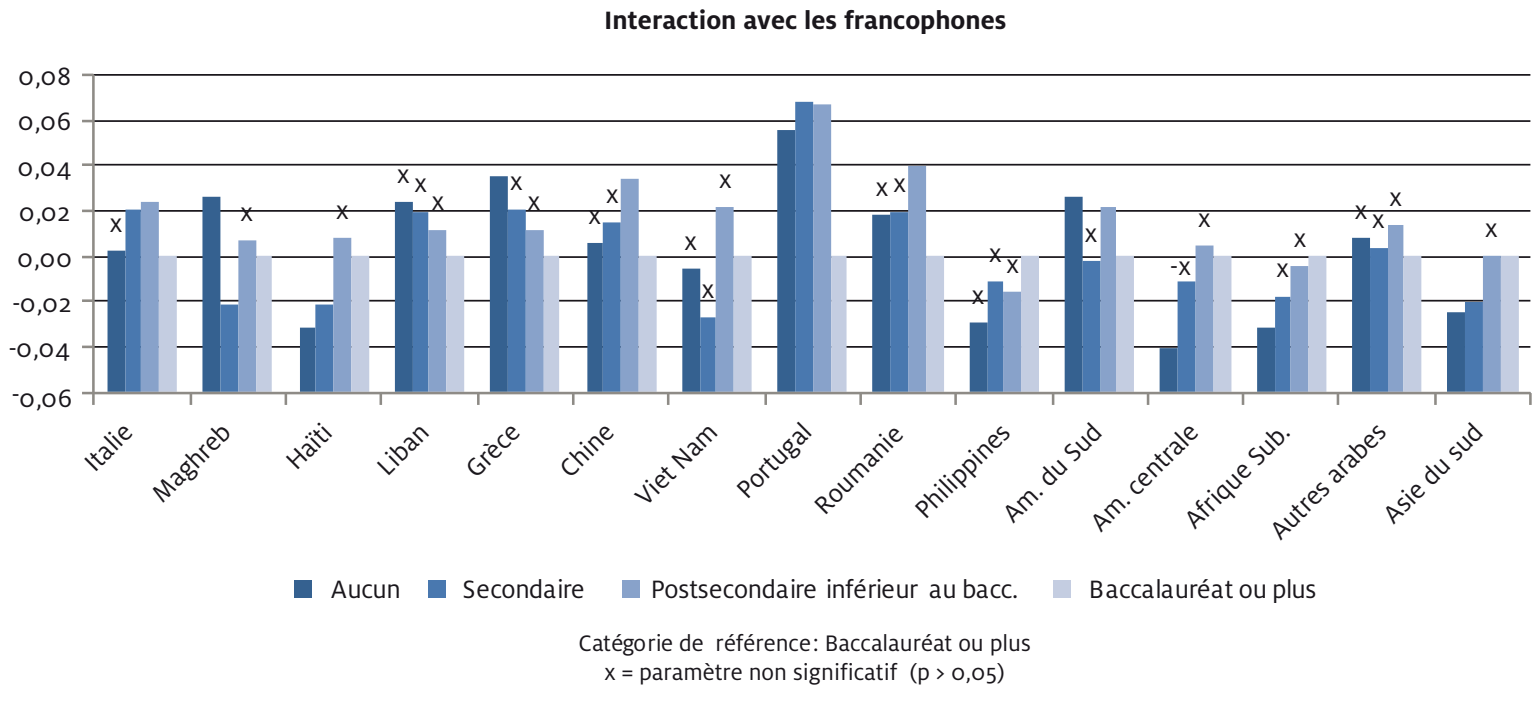

Interaction avec les anglophones

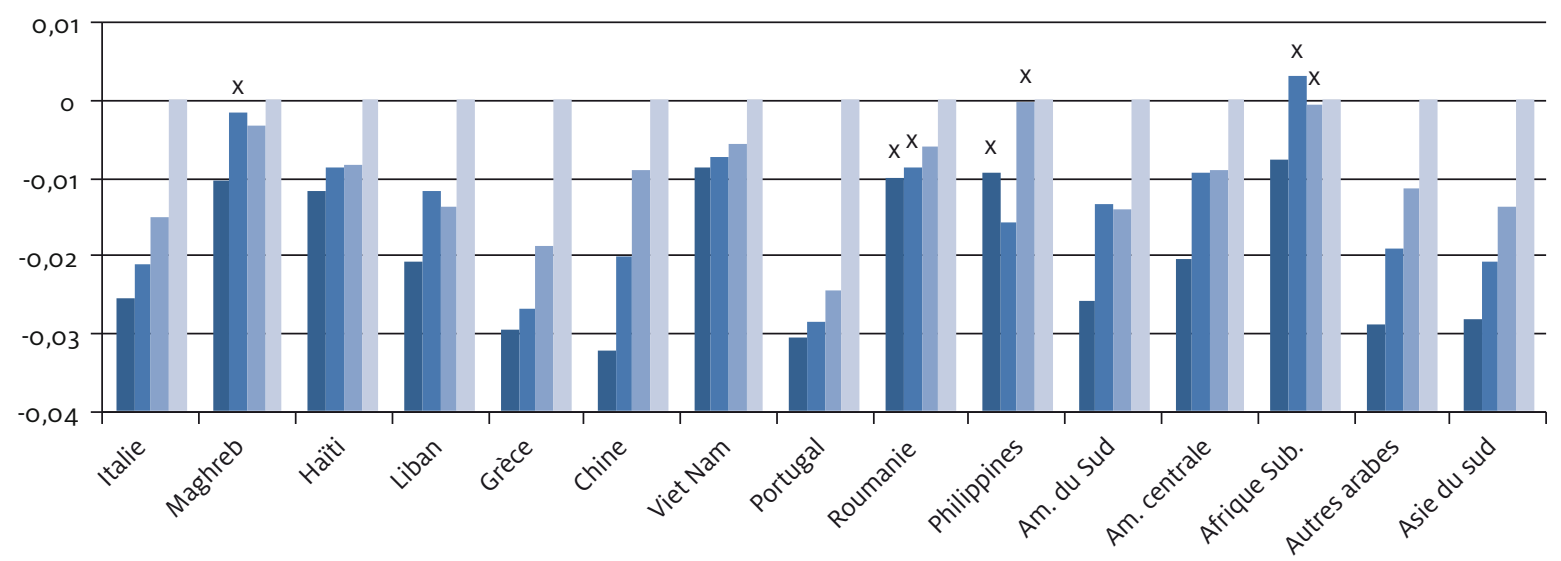

baccalauréat ou plus, l'effet de la scolarité est particulièrement marqué pour plusieurs communautés non francotropes tels que les Sud-Asiatiques, les Chinois, les Grecs et les Arabes d'autres pays. À l'opposé, les coefficients sont beaucoup plus faibles, parfois statistiquement non significatifs, pour certaines communautés francotropes telles que les Vietnamiens, les Subsahariens, les Haïtiens et les Maghrébins.

Concernant l'interaction résidentielle avec les francophones, l'effet de la scolarité est beaucoup moins évident, puisque la plupart des paramètres ne sont pas significatifs et lorsqu'ils le sont, aucune tendance précise ne ressort. Tout au plus, une meilleure éducation semble associée à une plus forte propension à vivre parmi les francophones seulement pour les communautés haïtienne, centre-américaine, subsaharienne et sud-asiatique. Pour les autres communautés immigrantes, les coefficients ne sont généralement pas significatifs, à l'exception des communautés portugaises où 
une nette démarcation s'observe entre ceux qui ont un baccalauréat et plus et les autres, qui ont une plus forte propension à l'exposition aux francophones.

Pour terminer, les modèles intègrent aussi une variable liée à la connaissance des langues officielles. Sans surprise, l'effet est tel qu'attendu pour toutes les communautés, à savoir que la connaissance d'une langue tend à favoriser la propension à vivre dans un secteur où cette langue est aussi parlée. La structure familiale n'a quant à elle pas d'effet décelable.

\section{DISCUSSION ET CONCLUSION}

Dans cette étude, nous cherchions à mesurer les préférences en matière de composition linguistique du voisinage des principales communautés immigrantes de Montréal. Puisque les communautés immigrantes sont très hétérogènes du point de vue de leurs caractéristiques socioéconomiques et de leurs parcours migratoires, nous avons développé des modèles statistiques permettant d'exercer un contrôle statistique sur la composition des groupes de manière à mesurer les comportements spécifiques des différentes communautés.

Il en ressort en premier lieu que dans l'ensemble, aucune communauté n'est complètement isolée du groupe francophone ou du groupe anglophone. Pour toutes les communautés, tant les indicateurs d'interaction bruts que standardisés montrent qu'une proportion moyenne appréciable de francophones ou d'anglophones habitent les mêmes quartiers. Cependant, considérant que le poids démographique des francophones est nettement supérieur à celui des anglophones, les indicateurs d'interaction modifiés montrent des différences importantes dans la surexposition ou la sous-exposition des communautés immigrantes aux deux communautés natives. Les communautés francotropes sont soit exposées normalement à celles-ci (par exemple, les Portugais et les Sud-Américains), soit sous-exposées aux deux (par exemple, les Vietnamiens, les Maghrébins et les Haïtiens). Les communautés non francotropes sont quant à elles toutes nettement sousexposées aux francophones, notamment les Grecs, Philippins et Sud-Asiatiques, et plusieurs sont surexposés aux anglophones. En somme, l'assimilation spatiale des communautés immigrantes ne se fait souvent pas en référence aux francophones majoritaires, mais plutôt en référence à la minorité anglophone. Considérant la forte relation qui existe entre la langue du voisinage et l'intégration linguistique (Bourbeau et collab., 2011; Carpentier, 2004), les choix résidentiels de ces communautés ne favorisent probablement pas leur francisation tel que voulu par les politiques d'intégration.

La sous-exposition aux francophones observée pour certaines communautés immigrantes cache parfois une forte hétérogénéité de comportements. En additionnant l'indice d'interaction standardisé (l'ordonnée à l'origine des régressions) aux paramètres associés à certaines caractéristiques, nous obtenons des indices d'interaction résidentielle avec les francophones spécifiques pour ces sous-groupes. Parmi ces communautés sous-exposées, certains sous-groupes présentant un certain nombre de caractéristiques le sont encore plus, au point où leur interaction résidentielle avec les francophones est fortement limitée. C'est le cas par exemple des Philippins (indice standardisé de 0,284$)$ du premier quintile de revenu (-0,049), qui connaissent seulement l'anglais $(-0,061)$ et qui sont arrivés entre 2006 et $2011(-0,047)$, où la somme des paramètres donne un indice de 0,127 , signifiant qu'en moyenne, il n'y a que $12,7 \%$ de francophones dans les quartiers de 
résidence de ce sous-groupe particulier. En normalisant pour le poids démographique des francophones, nous obtenons un indice modifié de 0,233 , ce qui signifie que les francophones sont quatre fois moins nombreux dans leurs quartiers de résidence que ce qui serait attendu s'il y avait répartition égale de la population. Ce profil particulier semble de surcroît correspondre aux personnes ayant immigré par le programme des aides familiales résidantes pour travailler au domicile d'employeurs fortunés généralement anglophones (Dubreuil et Marois, 2011). En retour, une certaine frange de la communauté philippine n'est pas du tout sous-exposée, soit celle de la $2^{\mathrm{e}}$ génération dont un des deux parents est né au Canada $(+0,260)$. Plus globalement, il est intéressant de noter que la deuxième génération issue d'un couple formé d'un parent natif et d'un parent immigrant favorise significativement et fortement l'interaction résidentielle avec la majorité francophone pour presque tous les groupes. Bon nombre de ces unions sont des unions formées au Québec. Il pourrait donc exister une corrélation entre la formation d'unions au Québec et l'intégration spatiale avec la majorité francophone. En outre, ces observations démontrent qu'il est nécessaire de décomposer les populations non seulement selon leur origine, mais aussi selon leurs caractéristiques socioéconomiques lorsque l'on cherche à déceler les différentes dynamiques résidentielles.

Les modèles de régression ont ainsi permis de constater que certains sous-groupes ont des comportements nettement différents des autres. Pour la plupart des communautés immigrantes, une tendance générale peut être décelée : les membres les mieux nantis en termes de revenu et d'éducation ont une propension beaucoup plus forte à vivre avec les anglophones. La classe moyenne est quant à elle plus portée à vivre avec les francophones, alors que les plus pauvres sont plus propices à l'isolement résidentiel ou à vivre dans des quartiers multiethniques. L'élite économique étant traditionnellement anglophone et les francophones étant dans une position infériorisée (Brunet, 1955 ; Séguin, 1987 ; Vaillancourt, 1985), ces observations concernant les communautés immigrantes tendent à montrer que ces dernières reproduisent en quelque sorte la dynamique de ségrégation spatiale entre les francophones et anglophones selon leur classe sociale. Le « place stratification model » observé dans plusieurs métropoles américaines qui contribue à maintenir les ségrégations entre les Blancs, les Afro-Américains et Hispaniques pourrait possiblement se transposer à Montréal, mais sur le plan linguistique plutôt qu'ethnique. Cette hypothèse corrobore par ailleurs les observations de Marois et Bélanger (2014b) sur l'absence de « White Flight » à Montréal (c'est-à-dire un exode des populations blanches), phénomène en retour remplacé par un « French Flight».

Pour terminer, rappelons que la perspective transversale utilisée dans cette étude limite les interprétations possibles des variables temporelles telles que l'âge ou la durée de résidence. Cette approche ne permet en effet pas de distinguer les changements de comportement intergénérationnels des changements intragénérationnels. La transformation des questionnaires détaillés des recensements en enquête non obligatoire en 2011 rend toutefois hasardeux le suivi des cohortes dans le temps étant donné le biais probable de non-réponses. De toute évidence, il y aurait un besoin pour des données longitudinales exhaustives pour pousser l'analyse de l'intégration spatiale des immigrants au Canada. 


\section{BIBLIOGRAPHIE}

Alba, R. D. et J. Logan. 1992. " Analyzing Locational Attainments : Constructing Individual-Level Regression Models Using Aggregate Data », Sociological Methods \& Research, 20, 3 : 367-397.

Andersen, H. S. 2010. "Spatial Assimilation in Denmark? Why do Immigrants Move to and from Multi-ethnic Neighbourhoods?», Housing Studies, 25, $3:$ 281-300.

Apparicio, P. 2000. "Les indices de ségrégation résidentielle : un outil intégré dans un système d'information géographique », Cybergeo : European Journal of Geography, 134.

Apparicio, P., X. Leloup et P. Rivet. 2006. La répartition spatiale des immigrants à Montréal : apport des indices de ségrégation résidentielle. Centre de recherche interuniversitaire de Montréal sur l'immigration, l'intégration et la dynamique urbaine.

Apparicio, P. et A. M. SÉguin. 2002. «La division de l'espace résidentiel montréalais en fonction de la langue maternelle : apport des indices de ségrégation résidentielle », Canadian Journal of Urban Research, 11, $2: 265-297$.

Bélanger, A., R. Lachapelle et P. Sabourin. 2011. Persistance et orientation linguistiques de divers groupes d'allophones au Québec, Portrait démolinguistique, Office québécois de la langue française.

Bélanger, A., P. Sabourin et R. Lachapelle. 2011. "Une analyse des déterminants de la mobilité linguistique intergénérationnelle des immigrants allophones au Québec », Cahiers québécois de démographie, 40, $1: 113-138$.

Bell, W. 1954. "A Probability Model for the Measurement of Ecological Segregation », Social Forces, 32, $4: 357-364$.

Bohnert, N., J. Chagnon et P. Dion. 2015. Projections démographiques pour le Canada (2013 à 2063), les provinces et les territoires (2013 à 2038). Ottawa : Statistique Canada.

Bourbeau, R., N. Robitaille et R. Ouellet. 2011. Caractéristiques du quartier de résidence et comportements linguistiques des immigrants allophones de la RMR de Montréal. In Portrait démolinguistique : Office québécois de la langue française.

Brown, L. A. et S.-Y. Chung. 2006. "Spatial segregation, segregation indices and the geographical perspective », Population, Space and Place, 12, $2: 125-143$.

Brown, L. A. et S.-Y. Chung. 2008. " Market-Led Pluralism : Rethinking Our Understanding of Racial/Ethnic Spatial Patterning in U.S. Cities », Annals of the Association of American Geographers, 98, $1: 180-212$.

Brunet, M. 1955. Canadians et Canadiens. Étude sur l'histoire et la pensée des deux Canada. Montréal et Paris, Fides.

CArpentier, A. 2004. Tout est-il joué avant l'arrivée ? Étude de facteurs associés à un usage prédominant du français ou de l'anglais chez les immigrants allophones arrivés au Québec adultes. Conseil supérieur de la langue française.

Charles, C. Z. 2003. « The Dynamics of Racial Residential Segregation », Annual Review of Sociology, 29, $1: 167-207$.

Chung, S.-Y. et L. A. Brown. 2007. «Racial/Ethnic Residential Sorting in Spatial Context : Testing the Explanatory Frameworks1 », Urban Geography, 28, 4:312-339. 
Cort, D. A. 2011. « Reexamining the ethnic hierarchy of locational attainment : Evidence from Los Angeles », Social Science Research, 40, $6: 1521-1533$.

Dubreuil, B. et G. Marois. 2011. «Une pénurie de domestique » éd. Le remède imaginaire. Pourquoi l'immigration ne sauvera pas le Québec. Montréal, Boréal.

Fitoussi, J.-P., É. LAurent et M. JoËL. 2004. Ségrégation urbaine et intégration sociale. Paris, La Documentation française.

Fong, E. et M. Gulia. 1999. «Differences in Neighborhood Qualities among Racial and Ethnic Groups in Canada », Sociological Inquiry, 69, $4: 575-598$.

Frey, W. H. 2006. America's Regional Demographics in the 'oos Decade: The Role of Seniors, Boomers and New Minorities. Research Institute for Housing America Research Paper

Germain, A., D. Rose et M. Richard. 2012. «Les banlieues de l'immigration ou quand les immigrants refont les banlieues », dans D. Fougère, éd. Histoire de Montréal et de sa région. Québec, Presses de l'Université Laval : 1107-1142.

Glikman, A. et M. Semyonov. 2012. «Ethnic Origin and Residential Attainment of Immigrants in European Countries », City \& Community, 11, 2 : 198-219.

Gouvernement Du QuéBec. 2015. Politique québécoise en matière d'immigration, de participation et d'inclusion. Ensemble, nous sommes le Québec. sous la dir. de D. L. D. E. D. L. I. MinisTÈRE DE L'IMMIGRATION.

Greene, W. H. 1997. Econometric Analysis. Upper Saddle River, N.J., Prentice Hall.

Institut De La STATistiQue DU QUéBec. 2015. Migrations internationales et interprovinciales, Québec, par région métropolitaine, 2001-2014.

Karsten, S., C. Felix, G. Ledoux, W. Meijnen, J. Roeleveld et E. Van Schooten. 2006. "Choosing Segregation or Integration?: The Extent and Effects of Ethnic Segregation in Dutch Cities », Education and Urban Society, 38, $2: 228-247$.

Logan, J., R. D. Alba, T. Mcnulty et B. Fisher. 1996. « Making a Place in the Metropolis : Locational Attainment in Cities and Suburbs ", Demography, 33, $4: 443-453$.

Logan, J., W. Zhang et R. D. Alba. 2002. «Immigrant Enclaves and Ethnic Communities in New York and Los Angeles », American Sociological Review, 67, 2 : 299-322.

Marois, G. et A. BÉLAnger. 2014a. «De Montréal vers la banlieue : déterminants du choix du lieu de résidence », Cahiers québécois de démographie, 43, $2:$ 439-468.

Marois, G. et A. Bélanger. 2014b. « Déterminants de la migration résidentielle de la ville centre vers la banlieue dans la région métropolitaine de Montréal : clivage linguistique et fuite des francophones », The Canadian Geographer / Le Géographe canadien, 58, 2 : 141-159.

Marois, G. et S. Lord. 2017. «A Statistical Approach for Analyzing Residential Isolation and its Determinants for Immigrant Communities : an Application to the Montréal Metropolitan Region ", Applied Spacial Analysis and Policy, doi : https ://doi.org/10.1007/s12061-017-9223-9.

Massey, D. S. et N. A. Denton. 1985. "Spatial Assimilation as a Socioeconomic Outcome ", American Sociological Review, 50, $1:$ 94-106.

Myles, J. et F. Hou. 2003. Établissement réussi et ségrégation résidentielle parmi les minorités visibles de Toronto. Ottawa : Statistique Canada.

Paillé, M. 2000. « Migrations intérieures des Québécois d'expression française, 1981-1986 et 19861991 », Cahiers québécois de démographie, 29, 1 : 147-167. 
Park, R. E. et E. W. Burgess. 1925. The city. University of Chicago Press.

Pelletier, D. 2012. "Accéder au meilleur quartier possible : types de famille et ségrégation résidentielle croisée à Montréal », Cahiers québécois de démographie, 41, 2 : 257-298.

Rosenbaum, E. et S. Friedman. 2001. "Differences in the Locational Attainment of Immigrant and Native-Born Households with Children in New York City ", Demography, 38, 3: 337-348.

SÉguin, A.-M., D. Rose et J. Mongeau. 2003. L'insertion résidentielle des jeunes issus de l'immigration à Montréal, Rapport de recherche dans le cadre d'Immigration et métropoles, SCHL.

SÉguin, M. 1987. "Les normes », dans R. Comeau, éd. Maurice Séguin, historien du pays québécois vu par ses contemporains. Montréal, VLB : 81-220.

Sélimanovski, C. 2008. La frontière de la pauvreté. Rennes, Presses Universitaires de Rennes.

Shimadeh, E. S. et N. Flynn. 1996. "Segregation and Crime: The Effect of Black Social Isolation on the Rates of Black Urban Violence », Social Forces, 74, $4: 1325-1352$.

Statistique CANAda. 2011. Enquête nationale sur les ménages.

Statistique Canada. 2013. Enquête nationale auprès des ménages 2011. Ottawa, Statistique Canada.

Vaillancourt, F. 1985. Économie et langue : recueil de textes. Center de recherche en développement économique, Université de Montréal, $302 \mathrm{p}$.

Van Kempen, R. et A. Şule Özüekren. 1998. «Ethnic Segregation in Cities : New Forms and Explanations in a Dynamic World », Urban Studies, 35, 10 : 1631-1656.

Walton, E. 2012. «Resurgent Ethnicity among Asian Americans : Ethnic Neighborhood Context and Health », Journal of Health and Social Behavior, 53, 3:378-394.

Wen, M., D. S. Lauderdale et N. R. Kandula. 2009. "Ethnic Neighborhoods in Multi-Ethnic America, 1990-2000 : Resurgent Ethnicity in the Ethnoburbs? », Social Forces, 88, 1 : 425-460. 


\section{ANNEXE 1}

Paramètres des régressions sur la proportion de francophones vivant dans le quartier de résidence, région métropolitaine de Montréal, 2011 (1/2)

\begin{tabular}{|c|c|c|c|c|c|c|c|c|}
\hline & Italie & Maghreb & Haïti & Liban & Grèce & Chine & Viet Nam & Portugal \\
\hline Ordonnée à l'origine & $0,385 * * *$ & $0,378 * * *$ & $0,459 * \star \star$ & $0,365 * * *$ & $0,382 * * *$ & $0,400 * * *$ & $0,409 * * *$ & $0,504 * * *$ \\
\hline \multicolumn{9}{|l|}{ Sexe (réf = Homme) } \\
\hline Femme & 0,006 & $-0,001$ & 0,005 & $-0,007$ & $-0,010$ & $-0,009$ & 0,026 * & $-0,015$ \\
\hline \multicolumn{9}{|l|}{ Groupe d'âge (réf = 30-34) } \\
\hline Moins de 25 & $-0,153 * * *$ & $-0,025$ & $-0,021$ & $-0,028$ & $-0,031$ & $-0,082 * * *$ & 0,027 & $-0,066$ \\
\hline $25-29$ & $-0,051 * \star$ & 0,007 & $-0,021$ & $-0,037$ & $-0,046$ & $-0,015$ & 0,016 & $-0,046$ \\
\hline $35-39$ & $-0,009$ & 0,008 & 0,004 & 0,024 & $-0,003$ & $-0,020$ & 0,013 & $-0,004$ \\
\hline $40-44$ & $-0,012$ & 0,007 & 0,007 & $-0,028$ & $-0,059 * *$ & $-0,008$ & $-0,015$ & $-0,019$ \\
\hline $45-49$ & $-0,010$ & 0,025 * & $-0,014$ & $-0,029$ & $-0,057 * \star$ & $-0,014$ & $-0,016$ & $-0,071 * \star$ \\
\hline $50-54$ & $-0,034 * *$ & $-0,006$ & $-0,048 * \star *$ & $-0,040 *$ & $-0,054^{* *}$ & $-0,025$ & $-0,003$ & $-0,082 * *$ \\
\hline $60-65$ & $-0,020$ & $-0,018$ & $-0,038 * *$ & $-0,072 * \star$ & $-0,080 * *$ & $-0,042$ * & 0,009 & $-0,078 * *$ \\
\hline $65+$ & $-0,036 * *$ & $-0,100 * * *$ & $-0,050 * *$ & $-0,063 * *$ & $-0,076 * *$ & $-0,022$ & $-0,002$ & $-0,119 * * *$ \\
\hline \multicolumn{9}{|c|}{ Statut générationnel (réf = 1re génération, arrivé entre 1981 et 1990) } \\
\hline $1^{\text {re }}$ génération, arrivé avant 1971 & $-0,025$ & $-0,091 * * *$ & 0,060 ** & 0,006 & $-0,081 * \star$ & $-0,055^{* *}$ & $-0,131 *$ & 0,008 \\
\hline 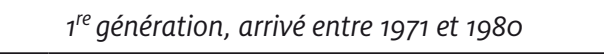 & $-0,017$ & $-0,045^{* *}$ & 0,016 & 0,008 & $-0,069 * *$ & $-0,037$ * & $-0,001$ & 0,024 \\
\hline $1^{\text {re }}$ génération, arrivé entre 1991 et 2000 & $0,082 * \star$ & $0,070^{\star * *}$ & $-0,026 * *$ & 0,011 & 0,001 & 0,020 & 0,001 & $-0,002$ \\
\hline $1^{\text {re }}$ génération, arrivé entre 2001 et 2005 & 0,067 & $0,052 * * *$ & 0,030 ** & $-0,015$ & 0,049 & 0,015 & 0,031 & $-0,161 * *$ \\
\hline 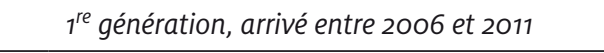 & 0,041 & $0,049^{* * *}$ & $-0,043^{* * *}$ & $-0,034$ * & 0,032 & $-0,026$ & 0,045 & $-0,014$ \\
\hline $2^{e}$ génération, deux parents nés à l'étranger & 0,003 & $-0,136 * \star *$ & 0,017 & 0,009 & $-0,036$ & $-0,062 * \star$ & $0,074 * \star$ & 0,001 \\
\hline $2^{e}$ génération, un seul parent né à l'étranger & $0,134^{* * *}$ & $0,139 * \star *$ & $0,072 * \star$ & $0,133^{\star * *}$ & $0,124 * * *$ & $0,1144^{\star *}$ & $0,167^{\star * *}$ & $0,124^{* * *}$ \\
\hline Résident non permanent & 0,007 & 0,044 * & $-0,049 * *$ & $-0,026$ & $-0,091$ & $-0,100 * * *$ & $-0,049$ & $-0,020$ \\
\hline
\end{tabular}


ANNEXE $1(1 / 2)$ (suite)

\begin{tabular}{|c|c|c|c|c|c|c|c|c|}
\hline & Italie & Maghreb & Haïti & Liban & Grèce & Chine & Viet Nam & Portugal \\
\hline \multicolumn{9}{|l|}{ Scolarité } \\
\hline Aucun & 0,002 & 0,026 * & $-0,031 * *$ & 0,024 & $0,035 * *$ & 0,006 & $-0,006$ & $0,055 * \star$ \\
\hline Secondaire & $0,021 * *$ & $-0,021 *$ & $-0,021 *$ & 0,020 & 0,020 & 0,015 & $-0,027$ & $0,068 * *$ \\
\hline Postsecondaire inférieur au bacc. & $0,024 * * *$ & 0,007 & 0,008 & 0,012 & 0,012 & 0,034 ** & 0,022 & 0,067 *** \\
\hline Anglais seulement & $-0,084^{* * *}$ & 0,051 & $-0,058$ & $-0,036 *$ & $-0,059^{* * *}$ & $-0,062 * * *$ & $-0,020$ & $-0,032$ \\
\hline Français seulement & $0,028 * * *$ & $0,027 * * *$ & $-0,006$ & $0,042 * * *$ & $0,154 * * *$ & 0,011 & 0,025 * & $0,058 * * *$ \\
\hline Aucune & $-0,043 * \star$ & 0,081 & $0,078 * \star$ & $-0,049$ & $-0,094^{\star \star *}$ & $-0,049^{* \star}$ & $-0,015$ & 0,058 * \\
\hline \multicolumn{9}{|l|}{ Quintile de revenu (réf $=3$ ) } \\
\hline Plus pauvre & $-0,010$ & $-0,080 * * *$ & $-0,038 * * *$ & $-0,035 * *$ & $-0,017$ & $-0,024 *$ & $-0,031 *$ & $-0,033 *$ \\
\hline 4 & 0,009 & 0,031 ** & $0,048 * * *$ & $-0,001$ & 0,029 ** & 0,000 & $-0,015$ & $-0,009$ \\
\hline Plus riche & $-0,002$ & 0,001 & $0,048 * * *$ & $-0,001$ & 0,020 & $-0,006$ & $-0,009$ & $-0,045^{* *}$ \\
\hline \multicolumn{9}{|c|}{ Structure familiale (réf = Couple sans enfant) } \\
\hline Couple avec enfant & $-0,024 * * *$ & $-0,026 * *$ & 0,013 & $-0,016$ & $-0,030 * *$ & $-0,021 *$ & $-0,021$ & 0,004 \\
\hline Famille monoparentale & $-0,017$ * & $-0,024$ & $-0,005$ & $-0,043$ * & $-0,023$ & $-0,001$ & $-0,071 * \star \star$ & $-0,030$ \\
\hline Autre & $-0,016 *$ & 0,001 & $-0,023$ & $-0,034$ * & $-0,013$ & $-0,021$ & $-0,044 *$ & $-0,045 *$ \\
\hline
\end{tabular}

${ }^{*} \mathrm{p}<0,05 ;{ }^{* \star} \mathrm{p}<0,01 ;{ }^{* \star *} \mathrm{p}<0,0001$

Source: Enquête nationale auprès des ménages de 2011, calculs des auteurs 


\section{ANNEXE 1}

Paramètres des régressions sur la proportion de francophones vivant dans le quartier de résidence, région métropolitaine de Montréal, 2011 (2/2)

\begin{tabular}{|c|c|c|c|c|c|c|c|c|c|c|c|c|c|}
\hline \multirow[b]{2}{*}{ Ordonnée à l'origine } & Roumanie & \multicolumn{2}{|c|}{ Philippines } & \multicolumn{2}{|c|}{$\begin{array}{l}\text { Amérique } \\
\text { du Sud }\end{array}$} & \multicolumn{2}{|c|}{$\begin{array}{l}\text { Amérique } \\
\text { centrale }\end{array}$} & \multicolumn{2}{|c|}{$\begin{array}{l}\text { Afrique } \\
\text { subsaha- } \\
\text { rienne }\end{array}$} & \multicolumn{2}{|c|}{$\begin{array}{l}\text { Autres pays } \\
\text { arabes }\end{array}$} & \multicolumn{2}{|c|}{$\begin{array}{c}\text { Asie } \\
\text { du Sud }\end{array}$} \\
\hline & $0,402 * \star \star *$ & 0,284 & $\star \star \star$ & $0,473 *$ & $\star \star \star *$ & 0,473 & $\star \star \star$ & 0,381 & $\star \star \star$ & 0,334 & $\star \star \star ~$ & 0,319 & $\star \star *$ \\
\hline \multicolumn{14}{|l|}{ Sexe (réf = Homme) } \\
\hline Femme & $-0,020$ & \multicolumn{2}{|l|}{$-0,006$} & \multicolumn{2}{|l|}{$-0,011$} & $-0,019$ & $\star \star$ & \multicolumn{2}{|l|}{$-0,009$} & \multicolumn{2}{|l|}{$-0,011$} & \multicolumn{2}{|l|}{0,009} \\
\hline \multicolumn{14}{|l|}{ Groupe d'âge (réf = 30-34) } \\
\hline Moins de 25 & $-0,018$ & \multicolumn{2}{|l|}{$-0,032$} & \multicolumn{2}{|l|}{$-0,004$} & \multicolumn{2}{|l|}{0,014} & \multicolumn{2}{|l|}{0,005} & \multirow{2}{*}{\multicolumn{2}{|c|}{\begin{tabular}{|l}
$-0,084 \quad * * *$ \\
$-0,029$
\end{tabular}}} & \multicolumn{2}{|l|}{$-0,023$} \\
\hline $25-29$ & $-0,046$ & \multicolumn{2}{|l|}{$-0,008$} & \multicolumn{2}{|l|}{$-0,020$} & \multicolumn{2}{|l|}{$-0,011$} & \multicolumn{2}{|l|}{0,037} & & & \multicolumn{2}{|l|}{$-0,009$} \\
\hline $35-39$ & $-0,009$ & \multicolumn{2}{|l|}{0,018} & 0,021 & & 0,000 & & 0,024 & * & $-0,028$ & * & 0,001 & \\
\hline $40-44$ & $-0,013$ & $-0,010$ & & 0,013 & & $-0,011$ & & 0,007 & & $-0,035$ & ** & $-0,021$ & \\
\hline $45-49$ & $-0,026$ & $-0,007$ & & $-0,011$ & & $-0,017$ & & 0,001 & & $-0,015$ & & $-0,035$ & $\star \star$ \\
\hline $50-54$ & $-0,049 *$ & 0,001 & & $-0,047 \times$ & & $-0,015$ & & 0,004 & & $-0,025$ & & $-0,023$ & \\
\hline $55-59$ & $-0,080 * *$ & $-0,057$ & $* *$ & $-0,062 *$ & $\star \star \star *$ & $-0,021$ & & $-0,029$ & & $-0,026$ & & $-0,038$ & ** \\
\hline $60-65$ & $-0,079 * *$ & 0,008 & & $-0,066 *$ & $\star * \star$ & $-0,025$ & & 0,015 & & $-0,055$ & ** & $-0,020$ & \\
\hline $65+$ & $-0,162 \quad * * \star$ & 0,004 & & $-0,075 *$ & $\star \star \star$ & $-0,021$ & & $-0,049$ & * & $-0,055$ & $\star * \star$ & $-0,006$ & \\
\hline Statut générationnel (réf $=1$ re génération, a & e 1981 et 199 & & & & & & & & & & & & \\
\hline $1^{r e}$ génération, arrivé avant 1971 & $-0,059$ & 0,040 & & $-0,039$ & & $-0,027$ & & 0,052 & & 0,005 & & $-0,026$ & \\
\hline 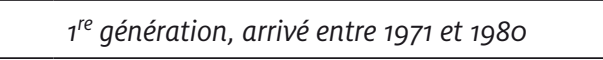 & $-0,053$ & $-0,008$ & & $0,033 *$ & ** & $-0,044$ & $\star \star \star$ & 0,014 & & 0,006 & & $-0,017$ & \\
\hline $1^{r e}$ génération, arrivé entre 1991 et 2000 & 0,017 & $-0,022$ & & $-0,019$ & & 0,017 & * & 0,000 & & $-0,002$ & & $-0,014$ & \\
\hline $1^{r e}$ génération, arrivé entre 2001 et 2005 & 0,032 & $-0,024$ & & $-0,023$ & & 0,013 & & 0,022 & & 0,000 & & $-0,019$ & \\
\hline $1^{r e}$ génération, arrivé entre 2006 et 2011 & $-0,063 * \star$ & $-0,047$ & $\star \star$ & $-0,034 *$ & ** & $-0,020$ & & $-0,030$ & * & $-0,004$ & & $-0,042$ & ** \\
\hline 2 génération, deux parents nés à l'étranger & $-0,102 \quad \star \star \star$ & 0,075 & * & $-0,015$ & & $-0,021$ & & 0,064 & * & 0,056 & $\star \star \star ~$ & 0,032 & \\
\hline 2 génération, un seul parent né à l'étranger & $-0,049$ & 0,260 & $\star \star \star$ & $0,057 *$ & $\star \star$ & 0,060 & $\star \star \star$ & 0,079 & $\star \star$ & 0,167 & $\star \star \star *$ & 0,126 & $\star \star \star *$ \\
\hline Résident non permanent & $-0,154 \quad * *$ & $-0,054$ & * & $-0,028$ & & $-0,038$ & $\star *$ & $-0,014$ & & $-0,065$ & ** & $-0,065$ & ** \\
\hline
\end{tabular}


ANNEXE $1(2 / 2)$ (suite)

\begin{tabular}{|c|c|c|c|c|c|c|c|}
\hline & Roumanie & Philippines & $\begin{array}{l}\text { Amérique } \\
\text { du Sud }\end{array}$ & $\begin{array}{l}\text { Amérique } \\
\text { centrale }\end{array}$ & $\begin{array}{l}\text { Afrique } \\
\text { subsaha- } \\
\text { rienne }\end{array}$ & $\begin{array}{c}\text { Autres pays } \\
\text { arabes }\end{array}$ & $\begin{array}{c}\text { Asie } \\
\text { du Sud }\end{array}$ \\
\hline \multicolumn{8}{|l|}{ Scolarité } \\
\hline Aucun & 0,018 & $-0,029$ & 0,026 * & $-0,040 * \star \star$ & $-0,031$ & 0,008 & $-0,025 *$ \\
\hline Secondaire & 0,019 & $-0,011$ & $-0,003$ & $-0,011$ & $-0,018$ & 0,004 & $-0,020 *$ \\
\hline Postsecondaire inférieur au bacc. & $0,040 * \star$ & $-0,016$ & $0,022 * *$ & 0,004 & $-0,004$ & 0,014 & 0,000 \\
\hline
\end{tabular}

Connaissance des langues officielles (réf = Anglais et français)

\begin{tabular}{|l|l|l|l|l|l|l|l}
\hline Anglais seulement & $-0,032$ & $-0,061 * * *$ & $-0,117 * * *$ & $-0,099 * * *$ & $-0,086 * * *$ & $-0,024 * *$ & $-0,039 * * *$ \\
\hline Français seulement & $0,075 * * *$ & 0,059 & $0,050 * * *$ & $0,024 * *$ & $0,051 \quad * * *$ & $0,069 * * *$ & $-0,035$ \\
\hline Aucune & 0,083 & 0,020 & $0,071 * *$ & $0,064 * * *$ & 0,111 & $-0,007$ \\
\hline
\end{tabular}

Quintile de revenu (réf $=3$ )

\begin{tabular}{|c|c|c|c|c|c|c|c|c|c|c|c|c|}
\hline Plus pauvre & $-0,048 * *$ & $-0,049$ & $\star \star \star *$ & $-0,037$ & $\star \star \star *$ & $-0,050$ & $* \star \star$ & $-0,059$ & $\star \star \star *$ & $-0,038$ & $\star \star \star$ & $-0,032 \quad * *$ \\
\hline 2 & $-0,022$ & $-0,055$ & $\star \star \star$ & $-0,035$ & $\star \star$ & $-0,027$ & ** & $-0,036$ & $\star \star$ & $-0,038$ & $* \star *$ & $-0,020 *$ \\
\hline 4 & 0,019 & 0,003 & & 0,013 & & 0,038 & $\star *$ & 0,072 & $\star \star \star *$ & $-0,013$ & & 0,023 \\
\hline Plus riche & $-0,021$ & 0,032 & * & $-0,009$ & & 0,012 & & 0,025 & & $-0,025$ & * & 0,012 \\
\hline \multicolumn{13}{|c|}{ Structure familiale (réf = Couple sans enfant) } \\
\hline Couple avec enfant & 0,019 & 0,004 & & $-0,005$ & & $-0,025$ & ** & 0,017 & & $-0,020$ & * & $-0,003$ \\
\hline Famille monoparentale & $-0,022$ & $-0,024$ & & $-0,030$ & * & $-0,052$ & $\star \star \star *$ & 0,010 & & $-0,027$ & * & $-0,018$ \\
\hline Autre & 0,003 & $-0,044$ & ** & $-0,031$ & ** & $-0,059$ & $* * *$ & $-0,011$ & & $-0,014$ & & $-0,029$ \\
\hline
\end{tabular}

${ }^{*} p<0.05 ;{ }^{* *} p<0.01 ; * * * p<0,0001$

Source: Enquête nationale auprès des ménages de 2011, calculs des auteurs 


\section{ANNEXE 2}

Paramètres des régressions sur la proportion d'anglophones vivant dans le quartier de résidence, région métropolitaine de Montréal, 2011 (1/2)

\begin{tabular}{|c|c|c|c|c|c|c|c|c|}
\hline & Italie & Maghreb & Haïti & Liban & Grèce & Chine & Viet Nam & Portugal \\
\hline Ordonnée à l'origine & $0,044^{* * *}$ & $0,055 * * *$ & $0,037^{* \star *}$ & $0,059 * * *$ & $0,061 * * *$ & $0,064^{* * *}$ & $0,041 * * *$ & $0,043 * * *$ \\
\hline \multicolumn{9}{|l|}{ Sexe (réf = Homme) } \\
\hline Femme & $-0,001$ & $0,005 * *$ & 0,001 & 0,000 & 0,001 & 0,005 * & $-0,002$ & $-0,003$ \\
\hline \multicolumn{9}{|l|}{ Groupe d'âge (réf = 30-34) } \\
\hline Moins de 25 & $0,022 * \star$ & 0,007 & 0,000 & 0,008 & $-0,001$ & $0,019 * \star$ & 0,000 & 0,003 \\
\hline $25-29$ & $-0,001$ & $-0,002$ & 0,004 * & 0,001 & $0,023 * \star$ & $-0,005$ & $-0,003$ & 0,002 \\
\hline $35-39$ & 0,004 & $-0,001$ & 0,002 & $-0,002$ & 0,000 & $0,010 *$ & 0,007 & $0,011 *$ \\
\hline $40-44$ & 0,006 * & $-0,004 *$ & 0,004 * & 0,004 & $0,014 * *$ & $0,017 * \star \star$ & 0,008 * & $0,011 *$ \\
\hline $45-49$ & $0,015 * * *$ & 0,002 & 0,002 & 0,003 & $0,021 * * *$ & $0,018 * * \star$ & 0,006 & $0,020 * * *$ \\
\hline $50-54$ & $0,019 * \star \star$ & 0,002 & $0,005^{* *}$ & 0,001 & $0,019 * *$ & $0,016 * *$ & 0,007 & $0,018 * *$ \\
\hline $55-59$ & $0,018 * * *$ & 0,004 & 0,003 & 0,004 & $0,023 * * *$ & 0,014 * & 0,001 & $0,016 * *$ \\
\hline $60-65$ & $0,021 \quad * * *$ & 0,001 & 0,003 & 0,011 * & $0,024 * * *$ & 0,014 * & 0,011 * & $0,017 * \star$ \\
\hline $65+$ & $0,020 * * *$ & 0,007 * & 0,004 * & 0,004 & $0,023 * * *$ & 0,012 * & 0,008 & $0,032 * * *$ \\
\hline \multicolumn{9}{|c|}{ Statut générationnel (réf = 1re génération, arrivé entre 1981 et 1990) } \\
\hline $1^{\text {re }}$ génération, arrivé avant 1971 & $-0,004$ & $0,021 * * *$ & $-0,001$ & 0,010 & $-0,004$ & 0,003 & $0,039 * * *$ & 0,004 \\
\hline $1^{\text {re }}$ génération, arrivé entre 1971 et 1980 & $-0,004$ & $0,021 * * *$ & $-0,001$ & 0,001 & $-0,005$ & $-0,001$ & $0,006 * \star$ & $-0,003$ \\
\hline 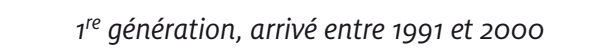 & 0,009 & $-0,014 * * *$ & $-0,003 * *$ & $-0,012 * * *$ & $-0,006$ & $-0,002$ & $-0,009 * *$ & $-0,007$ \\
\hline $1^{\text {re }}$ génération, arrivé entre 2001 et 2005 & 0,021 & $-0,018 * * *$ & $-0,004$ * & $-0,009 * *$ & 0,012 & $-0,001$ & 0,007 & 0,006 \\
\hline $1^{r e}$ génération, arrivé entre 2006 et 2011 & 0,024 * & $-0,020 * * *$ & $-0,001$ & $-0,007 *$ & 0,006 & $0,015 * *$ & 0,001 & 0,011 \\
\hline $2^{e}$ génération, deux parents nés à l'étranger & 0,004 & $0,043 * * *$ & 0,003 & 0,003 & 0,006 & $0,012 *$ & 0,005 & 0,006 \\
\hline $2^{e}$ génération, un seul parent né à l'étranger & $0,014 * *$ & 0,004 & $0,015 * \star \star$ & 0,008 & 0,014 * & 0,015 & 0,009 & 0,013 * \\
\hline Résident non permanent & 0,006 & $-0,013 * *$ & 0,001 & $-0,002$ & $0,061 * * *$ & 0,014 * & $-0,010$ & $-0,010$ \\
\hline
\end{tabular}


ANNEXe $2(1 / 2)$ (suite)

\begin{tabular}{|c|c|c|c|c|c|c|c|c|}
\hline & Italie & Maghreb & Haïti & Liban & Grèce & Chine & Viet Nam & Portugal \\
\hline \multicolumn{9}{|l|}{ Scolarité } \\
\hline Aucun & $-0,025^{* \star *}$ & $-0,010 * * *$ & 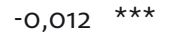 & $-0,021 \quad * \star *$ & $-0,029 * * *$ & $-0,032 * \star *$ & $-0,009 * *$ & $-0,030 * * *$ \\
\hline Secondaire & $-0,021 * * \star$ & $-0,002$ & $-0,009 * \star \star$ & $-0,012 * \star \star *$ & $-0,027 * \star \star$ & $-0,020 * \star \star$ & $-0,007$ * & $-0,029 * * *$ \\
\hline Postsecondaire inférieur au bacc. & $-0,0155^{\star \star \star}$ & $-0,003 * \star$ & $-0,008^{\star \star \star}$ & $-0,014{ }^{\star \star \star}$ & $-0,019 \star \star \star *$ & $-0,009 * \star$ & $-0,006$ * & $-0,025^{\star \star \star}$ \\
\hline \multicolumn{9}{|c|}{ Connaissance des langues officielles (réf = Anglais et français) } \\
\hline Anglais seulement & $0,033^{\star * *}$ & $0,019 * *$ & 0,006 & $0,012 \star \star *$ & $-0,004$ & $0,013^{* \star *}$ & 0,009 ** & 0,007 \\
\hline Français seulement & $-0,015 * * *$ & $-0,009 * * \star$ & $-0,006 * * \star$ & $-0,011 * * *$ & $-0,019 *$ & $-0,017 * \star$ & $-0,011 \quad * \star *$ & $-0,017 * * *$ \\
\hline Aucune & $-0,003$ & 0,002 & $-0,009$ * & $-0,011$ & 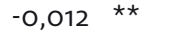 & $-0,003$ & $-0,005$ & $-0,009$ \\
\hline \multicolumn{9}{|l|}{ Quintile de revenu (réf $=3$ ) } \\
\hline Plus pauvre & 0,004 * & $-0,004$ * & $-0,003$ * & $-0,004$ & $-0,001$ & $-0,006$ * & $-0,003$ & $0,014 * \star \star$ \\
\hline 2 & 0,001 & $-0,002$ & $-0,003$ * & $-0,003$ & $-0,007^{*}$ & $-0,010 * *$ & $-0,004$ & $0,008 * \star$ \\
\hline 4 & $-0,001$ & 0,002 & 0,001 & 0,002 & 0,000 & 0,002 & 0,003 & 0,004 \\
\hline Plus riche & $0,011 * * *$ & $0,018 * * *$ & 0,004 * & $0,013^{* * *}$ & $0,012 * * *$ & 0,007 & 0,005 & $0,013 * *$ \\
\hline \multicolumn{9}{|c|}{ Structure familiale (réf = Couple sans enfant) } \\
\hline Couple avec enfant & 0,000 & 0,001 & $-0,002$ & $-0,002$ & $-0,004$ & $-0,003$ & $-0,007 *$ & 0,005 \\
\hline Famille monoparentale & $-0,001$ & $0,009 * *$ & $-0,002$ & $-0,006$ & $-0,004$ & $-0,003$ & 0,002 & $0,015 * *$ \\
\hline Autre & 0,002 & $-0,002$ & $-0,001$ & 0,002 & $-0,001$ & 0,001 & 0,001 & 0,009 * \\
\hline
\end{tabular}

${ }^{\star} \mathrm{p}<0, .05 ;{ }^{* \star} \mathrm{p}<0,01$; *** $\mathrm{p}<0,0001$

Source: Enquête nationale auprès des ménages de 2011, calculs des auteurs 


\section{ANNEXE 2}

Paramètres des régressions sur la proportion d'anglophones vivant dans le quartier de résidence, région métropolitaine de Montréal, 2011 (2/2)

\begin{tabular}{|c|c|c|c|c|c|c|c|c|c|c|c|c|c|}
\hline \multirow[b]{2}{*}{ Ordonnée à l'origine } & Roumanie & \multicolumn{2}{|c|}{ Philippines } & \multicolumn{2}{|c|}{$\begin{array}{l}\text { Amérique } \\
\text { du Sud }\end{array}$} & \multicolumn{2}{|c|}{$\begin{array}{l}\text { Amérique } \\
\text { centrale }\end{array}$} & \multicolumn{2}{|c|}{$\begin{array}{l}\text { Afrique } \\
\text { subsaha- } \\
\text { rienne }\end{array}$} & \multicolumn{2}{|c|}{$\begin{array}{c}\text { Autres pays } \\
\text { arabes }\end{array}$} & \multicolumn{2}{|c|}{$\begin{array}{l}\text { Asie } \\
\text { du Sud }\end{array}$} \\
\hline & $0,058 * * *$ & 0,071 & 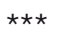 & 0,049 & $\star \star \star$ & 0,043 & $\star \star \star ~$ & 0,067 & $\star \star \star$ & 0,063 & $\star \star \star$ & 0,086 & *** \\
\hline \multicolumn{14}{|l|}{ Sexe (réf = Homme) } \\
\hline Femme & $0,011 \quad * \star$ & $-0,006$ & & 0,002 & & 0,006 & ** & 0,005 & * & 0,001 & & 0,007 & * \\
\hline \multicolumn{14}{|l|}{ Groupe d'âge (réf=30-34) } \\
\hline Moins de 25 & $-0,021$ & 0,000 & & 0,005 & & 0,004 & & 0,006 & & 0,020 & $\star \star$ & 0,006 & \\
\hline $25-29$ & $-0,001$ & 0,001 & & 0,000 & & $-0,001$ & & 0,002 & & 0,006 & & $-0,004$ & \\
\hline $35-39$ & 0,006 & 0,005 & & $-0,001$ & & 0,006 & * & 0,008 & ** & 0,012 & $\star \star$ & $-0,005$ & \\
\hline $40-44$ & 0,002 & 0,013 & * & 0,001 & & 0,008 & ** & 0,011 & $\star \star \star ~$ & 0,013 & $\star \star$ & $-0,006$ & \\
\hline $45-49$ & 0,000 & 0,007 & & 0,011 & $\star \star$ & 0,012 & $\star \star \star *$ & 0,010 & ** & 0,004 & & 0,003 & \\
\hline $50-54$ & 0,006 & $-0,001$ & & 0,013 & $\star \star \star *$ & 0,012 & $\star \star \star *$ & 0,011 & ** & 0,016 & $\star \star$ & $-0,002$ & \\
\hline $55-59$ & 0,015 * & 0,005 & & 0,015 & $\star * *$ & 0,019 & $\star \star \star *$ & 0,016 & $\star \star \star *$ & 0,001 & & $-0,003$ & \\
\hline $60-65$ & 0,004 & 0,007 & & 0,018 & $\star * *$ & 0,013 & ** & 0,011 & * & 0,003 & & $-0,004$ & \\
\hline $65+$ & 0,017 * & 0,005 & & 0,029 & $\star \star * *$ & 0,020 & $\star \star \star \star$ & 0,020 & $\star \star \star *$ & 0,003 & & 0,007 & \\
\hline \multicolumn{14}{|c|}{ Statut générationnel (réf = 1re génération, arrivé entre 1981 et 1990) } \\
\hline $1^{\text {re }}$ génération, arrivé avant 1971 & 0,017 & 0,019 & & 0,023 & $\star \star *$ & 0,018 & 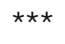 & 0,010 & & 0,019 & $* \star \star$ & 0,041 & *** \\
\hline 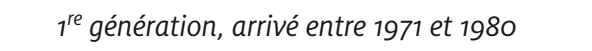 & $0,034 * * *$ & 0,022 & $\star \star$ & 0,004 & & 0,018 & $* * *$ & $-0,009$ & * & 0,008 & * & 0,013 & ** \\
\hline $1^{r e}$ génération, arrivé entre 1991 et 2000 & 0,007 & 0,007 & & 0,005 & & 0,005 & * & $-0,011$ & ** & $-0,001$ & & $-0,011$ & *** \\
\hline $1^{\text {re }}$ génération, arrivé entre 2001 et 2005 & $-0,003$ & $-0,004$ & & 0,013 & $\star * *$ & 0,011 & ** & $-0,008$ & * & $-0,007$ & & $-0,007$ & * \\
\hline $1^{r e}$ génération, arrivé entre 2006 et 2011 & 0,002 & $-0,005$ & & 0,011 & ** & 0,007 & ** & $-0,014$ & $\star \star \star *$ & $-0,003$ & & $-0,014$ & ** \\
\hline $2^{e}$ génération, deux parents nés à l'étranger & $0,048 * * *$ & 0,028 & ** & 0,019 & $\star \star \star *$ & 0,034 & $\star * *$ & 0,003 & & 0,002 & & 0,017 & ** \\
\hline $2^{e}$ génération, un seul parent né à l'étranger & $0,048 * * \star$ & 0,037 & * & 0,013 & * & 0,032 & $\star * \star$ & 0,015 & * & 0,002 & & 0,013 & \\
\hline Résident non permanent & 0,011 & $-0,009$ & & 0,009 & & 0,002 & & $-0,015$ & * & 0,001 & & $-0,001$ & \\
\hline
\end{tabular}


ANNEXE $2(2 / 2)$ (suite)

\begin{tabular}{|c|c|c|c|c|c|c|c|}
\hline & Roumanie & Philippines & $\begin{array}{l}\text { Amérique } \\
\text { du Sud }\end{array}$ & $\begin{array}{l}\text { Amérique } \\
\text { centrale }\end{array}$ & $\begin{array}{l}\text { Afrique } \\
\text { subsaha- } \\
\text { rienne }\end{array}$ & $\begin{array}{c}\text { Autres pays } \\
\text { arabes }\end{array}$ & $\begin{array}{c}\text { Asie } \\
\text { du Sud }\end{array}$ \\
\hline \multicolumn{8}{|l|}{ Scolarité } \\
\hline Aucun & $-0,010$ & $-0,009$ & $-0,026 * * *$ & $-0,020 * * *$ & $-0,007 *$ & $-0,029 \quad \star \star \star$ & $-0,028 * \star *$ \\
\hline Secondaire & $-0,009$ & $-0,016 \quad \star \star$ & $-0,013 * * *$ & $-0,009 * * *$ & 0,003 & $-0,019 \quad * * \star$ & $-0,021 * \star \star *$ \\
\hline Postsecondaire inférieur au bacc. & $-0,006 *$ & 0,000 & $-0,014 * * *$ & $-0,009 * * *$ & $-0,001$ & $-0,011 \quad \star \star \star$ & $-0,014 * * *$ \\
\hline
\end{tabular}

Connaissance des langues officielles (réf = Anglais et français)

\begin{tabular}{|c|c|c|c|c|c|c|c|c|c|c|c|}
\hline Anglais seulement & 0,013 * & 0,005 & 0,026 * & $\star \star \star ~$ & 0,024 & $\star \star \star$ & 0,037 & $\star \star \star *$ & 0,018 & $\star \star \star$ & 0,002 \\
\hline Français seulement & $-0,020 * * *$ & $-0,011$ & $-0,021 *$ & $\star \star \star ~$ & $-0,025$ & $\star \star \star *$ & $-0,010$ & 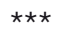 & $-0,024$ & $\star \star \star ~$ & $-0,009$ \\
\hline Aucune & 0,008 & $-0,036$ & $-0,029$ * & $\star \star \star ~$ & $-0,021$ & $\star \star \star$ & 0,007 & & 0,001 & & $-0,004$ \\
\hline
\end{tabular}

Quintile de revenu (réf $=3$ )

\begin{tabular}{|l|l|l|l|l|l|l|l|}
\hline Plus pauvre & $-0,001$ & $-0,017 * *$ & $-0,001$ & $-0,002$ & $-0,007 * *$ & $-0,001$ & $-0,013 * * *$ \\
\hline 2 & 0,000 & $-0,003$ & 0,002 & 0,004 & $-0,006 *$ & $-0,002$ \\
\hline 4 & $-0,006$ & 0,007 & 0,000 & 0,002 & $-0,006$ & $0,010 * *$ & $0,012 * *$ \\
\hline Plus riche & $0,012 * *$ & 0,001 & $0,012 * * *$ & $0,013 * * *$ & $0,007 *$ & $0,013 * * *$ & $0,018 * * *$ \\
\hline
\end{tabular}

Structure familiale (réf $=$ Couple sans enfant)

\begin{tabular}{|l|l|l|l|l|l|l|l|}
\hline Couple avec enfant & 0,006 & 0,001 & 0,004 & $0,005 *$ & $-0,005$ & 0,005 & 0,000 \\
\hline Famille monoparentale & $-0,008$ & 0,008 & 0,000 & 0,004 & $-0,010 * *$ & 0,008 & $-0,002$ \\
\hline Autre & $-0,001$ & $-0,003$ & $0,007 *$ & 0,004 & $-0,007 *$ & 0,001 & $-0,002$ \\
\hline
\end{tabular}

${ }^{*} \mathrm{p}<0,05 ;{ }^{* *} \mathrm{p}<0,01 ;{ }^{* \star *} \mathrm{p}<0,0001$

Source: Enquête nationale auprès des ménages de 2011, calculs des auteurs 